\title{
Thermodynamically stable asymptotically flat hairy black holes with a dilaton potential: the general case
}

\author{
Dumitru Astefanesei, ${ }^{a}$ Jose Luis Blázquez-Salcedo, ${ }^{b}$ Francisco Gómez $^{c}$ and \\ Raúl Rojas ${ }^{a}$ \\ ${ }^{a}$ Instituto de Física, Pontificia Universidad Católica de Valparaíso, \\ Casilla 4059, Valparaíso, Chile \\ ${ }^{b}$ Departamento de Física Teórica and IPARCOS, Universidad Complutense de Madrid, \\ E-28040 Madrid, Spain \\ ${ }^{c}$ Institute Lorentz of Theoretical Physics, Leiden University, \\ 2333 CA Leiden, The Netherlands \\ E-mail: dumitru.astefanesei@pucv.cl, jlblaz01@ucm.es, \\ f.j.gomez.serrano@umail.leidenuniv.nl, raul.rojas@pucv.cl
}

ABSTRACT: We extend the analysis, initiated in [1], of the thermodynamic stability of fourdimensional asymptotically flat hairy black holes by considering a general class of exact solutions in Einstein-Maxwell-dilaton theory with a non-trivial dilaton potential. We find that, regardless of the values of the parameters of the theory, there always exists a sub-class of hairy black holes that are thermodynamically stable and have the extremal limit well defined. This generic feature that makes the equilibrium configurations locally stable should be related to the properties of the dilaton potential that is decaying towards the spatial infinity, but behaves as a box close to the horizon. We prove that these thermodynamically stable solutions are also dynamically stable under spherically symmetric perturbations.

KEYwords: Black Holes, Black Holes in String Theory

ARXIV EPRINT: 2009.01854 


\section{Contents}

1 Introduction 2

2 Hairy black holes and their thermodynamics 4

2.1 Exact asymptotically flat solutions 4

2.2 Quasilocal formalism and energy 5

$\begin{array}{lll}2.3 & \text { First law of thermodynamics } & 6\end{array}$

$\begin{array}{lll}2.4 & \text { Quantum statistical relation } & 7\end{array}$

3 A general criterion for the local thermodynamic stability 8

3.1 Local thermodynamic stability conditions 8

$\begin{array}{ll}3.2 & \text { The general criterion }\end{array}$

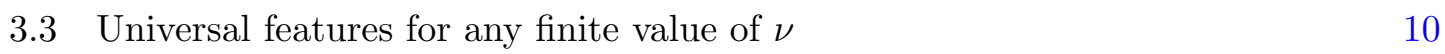

$\begin{array}{lll}3.3 .1 \quad \text { Grand-canonical ensemble, } T \text { and } \Phi \text { fixed } & 10\end{array}$

$\begin{array}{ll}\text { 3.3.2 Canonical ensemble, } T \text { and } Q \text { fixed } & 11\end{array}$

4 Comparison between hairy solutions with $\nu=3$ and $\nu=\infty \quad 14$

$\begin{array}{lll}4.1 & \text { Hairy black hole solution with } \nu=3 & 14\end{array}$

$\begin{array}{lll}4.2 & \text { Equation of state and critical points } & 15\end{array}$

$\begin{array}{lll}4.3 & \text { Thermodynamic stability in grand-canonical ensemble } & 17\end{array}$

$\begin{array}{ll}4.4 \text { Thermodynamic stability at } Q \text { fixed } & 18\end{array}$

$\begin{array}{ll}\text { 4.5 Extremality condition and the existence of a new branch } & 19\end{array}$

$\begin{array}{lll}5 & \text { Linear spherically symmetric perturbations } & 20\end{array}$

$\begin{array}{lll}6 & \text { Discussion } & 23\end{array}$

$\begin{array}{ll}\text { A Thermodynamic stability analysis for arbitrary } \nu & \mathbf{2 6}\end{array}$

A.1 Grand-canonical ensemble, $\Phi$ fixed 26

$\begin{array}{lll}\text { A.1.1 } Q \text { vs } \Phi, S \text { fixed diagram } & 27\end{array}$

$\begin{array}{ll}\text { A.1.2 } S \text { vs } T, \Phi \text { fixed diagram } & 27\end{array}$

$\begin{array}{ll}\text { A.1.3 Thermodynamic stability } & 28\end{array}$

A.2 Canonical ensemble, $Q$ fixed 28

A.2.1 $\Phi$ vs $Q, T$ fixed 28

$\begin{array}{lll}\text { A.2.2 } S \text { vs } T, Q \text { fixed } & 29\end{array}$

$\begin{array}{lll}\text { A.2.3 Thermodynamic stability } & 29\end{array}$ 


\section{Introduction}

With the discovery of black hole entropy [2] and Hawking radiation [3], the black hole physics provides a profound connection between thermodynamics and gravitation [4]. Although black hole thermodynamics is often studied with asymptotically flat boundary conditions, this is really not suitable for discussing equilibrium configurations. It is well-known that, e.g., Schwarzschild, Reissner-Nordström, and Kerr black holes are locally thermodynamically unstable.

An important question is, therefore, in which conditions there could exist thermodynamically stable black holes in flat spacetime? At first sight, this is not possible. To consistently put a black hole in thermal equilibrium with its Hawking radiation, we have to consider an indefinitely large reservoir of energy, which in turn implies that there is non-zero energy density out to infinity. One expects then a behaviour rather similar to a cosmological model that contracts or expands. Indeed, for the Schwarzschild black hole the heat capacity is negative and so a thermal fluctuation can break the equilibrium and that leads to the evaporation of the black hole or its indefinite growth. One way to circumvent this problem is to 'put the black hole in a box' [5]. ${ }^{1}$ Due to the fact that the temperature measured locally by a static observer is blue-shifted with respect to the usual temperature that is determined at asymptotically flat spatial infinity, the heat capacity becomes positive in a specific range of the box radius. Interestingly, there is another way to obtain thermodynamically stable asymptotically flat black holes without imposing artificial boundary conditions similar to the 'box' ones proposed by York in [5]. That is, one has to consider an obvious extension of the usual gravity models by considering scalar fields with self-interaction $[1,8,9]$. One of the main reasons to include this new sector in the action is coming from the fact that the scalar fields appear naturally as moduli in string theory.

The uniqueness theorem for the asymptotically flat, stationary black hole solutions of the Einstein-Maxwell equations is, by now, established quite rigorously. There also are several no-hair theorems in theories which couple scalar fields to gravity (see, e.g., [10] and references therein). However, in theories with a potential for the scalar field with certain properties $[11,12]$, there exist scalar-hairy black holes. For example, in asymptotically AdS spacetimes, various exact regular scalar-hairy black holes were analyzed in [13-22]. Interestingly, when the scalar fields are non-minimally coupled to gauge fields as in the models emerging from string theory, there exist a family of exact asymptotically flat hairy black holes [23-25] — in the context of no-hair theorems, the gauge field provides an effective potential for the scalar field and so the scalar field is not independent [26] (see, also, [27] for a recent discussion on dilatonic versus scalarised hairy black holes), the scalar charge is fixed by the other conserved charges.

In this paper we explore the thermodynamic and dynamic stability of a large class of exact asymptotically flat charged hairy black holes in a gravity model with a dilaton and its

\footnotetext{
${ }^{1}$ Another well known example is the anti-de Sitter (AdS) spacetime. In contrast with its asymptotically flat counterpart, asymptotically AdS spacetimes are not globally hyperbolic. The conformal asymptotic boundary at infinity is timelike and, in this case, the suitable initial data must be supplemented with appropriate boundary conditions [6]. Therefore, from a geometric standpoint, AdS spacetime behaves as a box $[7]$.
} 
potential [8]. We find a general criterion to obtain specific regions in parameter space where these black holes are thermodynamically stable and, then, we also show explicitly that they are dynamically stable under spherically symmetric perturbations. There is some previous related work [1,9] where a particular case was studied, though this case is obtained by rescaling the metric to remove a divergent factor and so it is not a generic representative of the general class of hairy black holes presented here.

It was shown in [28] that the dilaton potential of [8] emerges naturally in a consistent truncation of four-dimensional $N=2$ supergravity extended with vector multiplets and deformed by a dyonic Fayet-Iliopoulos (FI) term. This potential is characterized by three independent parameters: $\nu$ is a 'hairy' parameter that is related to the moduli metric of the model, $\alpha$ is related to the FI term, and the cosmological constant $\Lambda$. Its mathematical expression consists of two parts: one that is proportional with the cosmological constant and the other one is proportional to the parameter $\alpha$. In the limit $\Lambda \rightarrow 0$, the hairy solutions exist and they remain regular as in the AdS case. ${ }^{2}$

This model can be generalized by adding a gauge field to which the scalar field is coupled that provides an extension of the stringy model considered in [25] by including the dilaton potential. Some important properties of hairy black hole solutions in this extended model, when $\nu \rightarrow \infty$, were presented in [9] and we would like to briefly describe them here. Unlike the stringy solutions in [23-25], these new asymptotically flat solutions have a well defined extremal limit. However, unlike the Reissner-Nordström black hole, but similar to the solutions [23-25], they can be overcharged. Based on these features and a careful analysis of the dilaton potential, it was observed in [9] that, in fact, these solutions interpolate between Reissner-Nordström black hole and the stringy family of [23-25]. More importantly, there is a sub-class of thermodynamically stable hairy black holes [1] that are also dynamically stable. The dynamical robustness of these solutions was confirmed not just perturbatively, but also by a fully non-linear numerical simulations with the EinsteinMaxwell-dilaton system [9]. Since in this particular model the potential depends only on one parameter, $\alpha$, it is interesting to check how and if some of these properties are affected when the hairy parameter $\nu$ is turned on.

The remainder of the paper is organized as follows: in section 2, we present a general class of exact hairy black hole solutions and analyze their thermodynamic properties checking that the first law of thermodynamics and quantum statistical relation are consistently satisfied. In section 3, we obtain a general stability criterion based on the relative signs of the relevant response functions and prove that the thermodynamic behaviour is universal (for any value of $\nu$ ). We continue, in section 4, with a detailed study of a representative case with finite $\nu$ and compare the results with the case $\nu \rightarrow \infty$ studied previously in [1]. In section 5, we investigate the dynamical stability of hairy black holes solutions under spherically symmetric perturbations. We conclude in section 6 with a summary of our results and an extended discussion of the response functions in the particular case $\nu=3$. Finally, all the details regarding the regions of thermodynamic stability of the analysis in section 3 are presented in an appendix.

\footnotetext{
${ }^{2}$ The existence of asymptotically flat hairy solutions in theories with a potential for the scalar field was conjectured in [29], though the model is different (it can not be embedded in SUGRA) and the results are provided only numerically.
} 


\section{Hairy black holes and their thermodynamics}

In this section, we present a general family of exact asymptotically flat hairy electrically charged black hole solutions with a non-trivial scalar field potential [8]. We use the quasilocal formalism of Brown and York [30] supplemented with counterterms [31-35] to study their thermodynamics. We compute the quasilocal stress tensor [35], energy, on-shell Euclidean action (and the corresponding thermodynamic potential) and show that the first law of thermodynamics and quantum statistical relation are satisfied.

\subsection{Exact asymptotically flat solutions}

We will be studying the thermodynamic and dynamical stability in a theory where gravity couples to a dilaton, as well as the Maxwell field (with a dilaton potential, but vanishing cosmological constant),

$$
I=\frac{1}{2 \kappa} \int_{\mathcal{M}} d^{4} x \sqrt{-g}\left[R-\frac{1}{4} e^{\gamma \phi} F^{2}-\frac{1}{2}(\partial \phi)^{2}-V(\phi)\right]
$$

where the fundamental constants are set to $G_{N}=c=1(\kappa=8 \pi)$. As usual, the compact notation for the fields is $F^{2} \equiv F_{\mu \nu} F^{\mu \nu},(\partial \phi)^{2} \equiv g^{\mu \nu} \partial_{\mu} \phi \partial_{\nu} \phi$; the parameter $\gamma$ controls the strength of the coupling of the dilaton to the Maxwell field and, as we will see, it also determines the shape of the potential.

The equations of motion are

$$
\begin{aligned}
R_{\mu \nu}-\frac{1}{2} g_{\mu \nu} R & =T_{\mu \nu}^{\phi}+T_{\mu \nu}^{E M} \\
\partial_{\mu}\left(\sqrt{-g} e^{\gamma \phi} F^{\mu \nu}\right) & =0 \\
\frac{1}{\sqrt{-g}} \partial_{\mu}\left(\sqrt{-g} g^{\mu \nu} \partial_{\nu} \phi\right) & =\frac{d V(\phi)}{d \phi}+\frac{1}{4} \gamma e^{\gamma \phi} F^{2}
\end{aligned}
$$

where the corresponding energy-momentum tensors are $T_{\mu \nu}^{\phi} \equiv \frac{1}{2} \partial_{\mu} \phi \partial_{\nu} \phi-$ $\frac{1}{2} g_{\mu \nu}\left[\frac{1}{2}(\partial \phi)^{2}+V(\phi)\right]$ and $T_{\mu \nu}^{E M} \equiv \frac{1}{2} e^{\gamma \phi}\left(F_{\mu \alpha} F_{\nu}{ }^{\alpha}-\frac{1}{4} g_{\mu \nu} F^{2}\right)$. The general family of exact solution that we are going to consider was found in [8] for the following general selfinteracting potential

$$
V(\phi)=\frac{2 \alpha}{\nu^{2}}\left[\frac{\nu-1}{\nu+2} \sinh \left(\sqrt{\frac{\nu+1}{\nu-1}} \phi\right)-\frac{\nu+1}{\nu-2} \sinh \left(\sqrt{\frac{\nu-1}{\nu+1}} \phi\right)+4\left(\frac{\nu^{2}-1}{\nu^{2}-4}\right) \sinh \left(\frac{\phi}{\nu^{2}-1}\right)\right]
$$

where $\alpha$ is a real constant parametrizing the strength of the potential and $\nu$ is related to $\gamma$ by

$$
\gamma \equiv \sqrt{\frac{\nu+1}{\nu-1}}
$$

so that if $\nu \leq-1$ then $0 \leq \gamma \leq 1$ and, if $\nu \geq 1$ then $\gamma \geq 1$. The limit $\nu \rightarrow \infty$ corresponds to $\gamma=1$ and was studied in great detail in $[1,9]$. 
The equations of motion are solved by

$$
\begin{aligned}
d s^{2} & =\Omega(x)\left[-f(x) d t^{2}+\frac{\eta^{2} d x^{2}}{f(x)}+\left(d \theta^{2}+\sin ^{2} \theta d \varphi^{2}\right)\right], \\
A_{\mu} d x^{\mu} & =-\frac{q}{\nu x^{\nu}} d t, \quad \phi(x)=\sqrt{\nu^{2}-1} \ln (x)
\end{aligned}
$$

where the metric functions are

$$
\begin{aligned}
\Omega(x) & =\frac{\nu^{2} x^{\nu-1}}{\eta^{2}\left(x^{\nu}-1\right)^{2}}, \\
f(x) & =\frac{1}{\nu^{2}}\left[\alpha\left(\frac{x^{\nu+2}}{\nu+2}-x^{2}+\frac{x^{2-\nu}}{2-\nu}+\frac{\nu^{2}}{\nu^{2}-4}\right)+\eta^{2}\left(1-\frac{q^{2}}{2 \nu x^{\nu}} \frac{x^{\nu}-1}{\nu-1}\right)\left(x^{\nu}-1\right)^{2} x^{2-\nu}\right]
\end{aligned}
$$

With this special choice of the conformal factor $\Omega(x)$, the equation of motion for the dilaton can be easily integrated leading to a simple result. The constants $q$ and $\eta$ are the integral constants that define the conserved charges of the solutions. We notice that there is no integration constant related to the scalar field.

The exact solution presented so far is characterized by having two different branches of solutions, corresponding to the domains $x \in[0,1)$ and $x \in(1, \infty]$. The former is usually called the negative branch since the scalar field takes negative values, and the latter is called the positive branch. They actually correspond to two distinct families of solutions because the boundary condition at $x=1$ for the scalar field are different (for more details see [8]). In the remaining of the paper, we are going to present a detailed thermodynamic analysis only for the positive branch for which the thermodynamically stable black holes exist. ${ }^{3}$

\subsection{Quasilocal formalism and energy}

According to the formalism of Brown and York [30], the conserved quantities are obtained provided a hypersurface with an isometry generated by a Killing vector $\xi^{\mu}$ exists. All the observers living on this hypersurface measure the same conserved quantities. Using this specific foliation for the spacetime, the quasilocal stress tensor $\tau_{a b}$ can be defined as

$$
\tau_{a b}=\frac{2}{\sqrt{-h}} \frac{\delta I}{\delta h^{a b}}
$$

where $I=I_{b u l k}+I_{G H}+I_{c t}$ is the total action consisting of the bulk part of the action, given by (2.1), supplemented with the Gibbons-Hawking boundary term and the gravitational counterterm that cancels the infrared divergences of the theory. For asymptotically flat spacetimes in four dimensions, the gravitational counterterm is [31-33]

$$
I_{c t}=-\frac{1}{\kappa} \int_{\partial \mathcal{M}} d^{3} x \sqrt{-h} \sqrt{2 \mathcal{R}^{(3)}}
$$

\footnotetext{
${ }^{3}$ The negative branch contains only thermodynamically unstable black holes for any value of the parameter $\nu$ and that is why the thermodynamics of negative branch is not presented in what follows. This observation is also consistent with our previous work [1], where it was found that the black holes of negative branch, for the particular case of $\gamma=1$, are thermodynamically unstable.
} 
where $\mathcal{R}^{(3)}=h^{a b} \mathcal{R}_{a b}^{(3)}$ is the Ricci scalar on the boundary. We choose the foliation $x=$ const with the induced metric on each surface $h_{a b}$, whose trace is $h$. For the Killing vector $\xi=\partial / \partial t$, the conserved quantity is the total energy of the black hole (including the hair) [30]:

$$
E=\oint_{s_{\infty}^{2}} d^{2} \sigma \sqrt{\sigma} n^{a} \tau_{a t} \xi^{t}
$$

where $s_{\infty}^{2}$ is the spherical surface at infinity with $t=$ const, given by $d s^{2}=\sigma_{a b} d x^{a} d x^{b}$, where $n^{a}$ is the temporal unit normal vector. The concrete expression for the regularized quasilocal stress tensor in this case was obtained in [35]:

$$
\tau_{a b}=\frac{1}{\kappa}\left[K_{a b}-h_{a b} K+\left(\frac{1}{2} \mathcal{R}^{(3)}\right)^{-1 / 2}\left(\mathcal{R}_{a b}^{(3)}-h_{a b} \mathcal{R}^{(3)}\right)+h_{a b} \square \Psi-\Psi_{; a b}\right]
$$

where $\Psi=\sqrt{2 / \mathcal{R}^{(3)}} \cdot{ }^{4}$ By using the exact solution presented in the previous section, the non-zero components of the quasilocal stress tensor are

$$
\tau_{t t}=-\frac{(\Omega f)^{1 / 2}}{8 \pi \eta}\left(2 \eta f^{1 / 2}+\frac{\Omega^{\prime}}{\Omega} f\right), \quad \tau_{\theta \theta}=\frac{\tau_{\phi \phi}}{\sin ^{2} \theta}=\frac{\Omega^{1 / 2}}{8 \pi \eta f^{1 / 2}}\left(\frac{1}{2} f^{\prime}+\eta f^{1 / 2}+\frac{\Omega^{\prime}}{\Omega} f\right)
$$

Since the normal unit to $t=$ const can be written as $n_{a}=(f \Omega)^{1 / 2} \delta_{a}^{t}$, the total (conserved) energy is computed at the boundary $x=1$

$$
E=\frac{1}{2 \eta} \lim _{x \rightarrow 1}\left(2 \eta \Omega f^{1 / 2}+f \Omega^{\prime}\right)=\frac{q^{2}}{4 \eta(\nu-1)}-\frac{1}{6 \eta^{3}}\left(\alpha+3 \eta^{2}\right)
$$

It is straightforward to verify that, in this case, the conserved energy corresponds to the Arnowitt-Deser-Misner (ADM) mass obtained by expanding the $g_{t t}$ component in the canonical coordinates $[41-44] .^{5}$

\subsection{First law of thermodynamics}

Before computing the action on-shell, let us obtain the thermodynamic quantites for the solution presented in section 2.1. As explained before, we shall focus only on the positive branch, $x>1$.

The Hawking temperature is

$$
T=\frac{\eta\left(x_{+}^{\nu}-1\right)}{2 \pi \nu}\left[\frac{\left(x_{+}^{\nu}-1\right)}{4 \nu x_{+}^{\nu-1}}\left(\frac{3 q^{2}}{\nu-1}-\frac{2 \alpha}{\eta^{2}}+2 \nu-4\right)-\frac{q^{2}\left(x_{+}^{\nu}-1\right)^{2}}{2 \nu^{2} x_{+}^{2 \nu-1}}-x_{+}\right]
$$

where the horizon location is obtained from the horizon equation $f\left(x_{+}\right)=0$. The entropy is, as usual in gravity theories without higher derivative terms in the action, $S=A / 4=$

\footnotetext{
${ }^{4}$ This method was extensively used for various black hole/ring solutions [34-40].

${ }^{5}$ Since the asymptotics should be preserved, when the dilaton potential is non-trivial the asymptotic value of the scalar is fixed. However, when the dilaton can vary at the boundary, the total energy receives a new contribution $[45,46]$ and it does not match the ADM mass.
} 
$\pi \Omega\left(x_{+}\right)$, where $A$ is the area of the event horizon and $\Omega(x)$ is given by (2.9). The electric charge and its conjugate potential are

$$
Q \equiv \oint_{s_{\infty}^{2}} \star F=-\frac{q}{4 \eta}, \quad \Phi \equiv A_{t}(x=1)-A_{t}\left(x=x_{+}\right)=\frac{q}{\nu}\left(x_{+}^{-\nu}-1\right)
$$

where $F=\frac{1}{2} F_{\mu \nu} d x^{\mu} \wedge d x^{\nu}$ and $\star$ is the Hodge dual. Finally, the mass equals the conserved energy of the system, computed by the quasilocal formalism, $M=E$, where $E$ is given by the equation (2.16). It follows straightforwardly that they satisfy the first law of black hole thermodynamics

$$
d M=T d S+\Phi d Q
$$

with no independent contribution from the scalar field, which is secondary hair.

\subsection{Quantum statistical relation}

By taking the trace of the Einstein's equation (2.2) and replacing the Ricci scalar into the bulk part of the action, and by adding it to the Gibbons-Hawking boundary term, one gets that, on the Euclidean section, they add up to

$$
I_{b u l k}^{E}+I_{G H}^{E}=\beta(-S T-\Phi Q)-\frac{\beta}{\eta(x-1)}-\beta\left[\frac{2(\nu-1)\left(3 \eta^{2}+\alpha\right)-3 \eta^{2} q^{2}}{6 \eta^{3}(\nu-1)}\right]
$$

The gravitational counterterm contributes to the total action by

$$
I_{c t}^{E}=\beta M+\frac{\beta}{\eta(x-1)}+\beta\left[\frac{2(\nu-1)\left(3 \eta^{2}+\alpha\right)-3 \eta^{2} q^{2}}{6 \eta^{3}(\nu-1)}\right]
$$

and so the divergent term (and, also, the finite contribution) are canceled out. The total on-shell action satisfies the quantum statistical relation

$$
I^{E}=\beta(M-T S-\Phi Q) \equiv \beta \mathcal{G}
$$

where $\mathcal{G}=\mathcal{G}(T, \Phi)$ is the thermodynamic potential associated to the grand-canonical ensemble, where $\Phi$ is fixed as the consequence of the boundary condition $\left.\delta A_{\mu}\right|_{\partial \mathcal{M}}=0$.

In order to obtain the thermodynamic potential associated to the canonical ensemble, for which this time $Q$ is fixed as a consequence of the boundary condition $\left.\delta\left(e^{\gamma \phi} \star F\right)\right|_{\partial \mathcal{M}}=$ 0 , one would have to add a new boundary term to the action, $I \rightarrow I+I_{A}$, so that the action principle is well defined:

$$
I_{A}=\frac{1}{2 \kappa} \int_{\partial \mathcal{M}} d^{3} x \sqrt{-h} e^{\gamma \phi} n_{\mu} F^{\mu \nu} A_{\nu}
$$

The (geometrical) boundary term (2.23) in the action corresponds, from a thermodynamic point of view, to the Legendre transform from the grand-canonical ensemble to the canonical ensemble. The new contribution is $I_{A}^{E}=\beta Q \Phi$ and, therefore, the on-shell action for the canonical ensemble is

$$
I=\beta(M-T S) \equiv \beta \mathcal{F}
$$

where $\mathcal{F}=\mathcal{F}(T, Q)$ is the thermodynamic potential associated to the canonical ensemble. 


\section{A general criterion for the local thermodynamic stability}

In this section, we first review the conditions under which a black hole equilibrium configuration is thermodynamically stable against small fluctuations in canonical and grandcanonical ensemble. Then, we develop a consistent criterion to seek for stable configurations that is based on a study of relative signs of the response functions in the parameter space of the solutions. In section 3.2, we collect all the relevant definitions and make a summary with the main steps we are going to follow for the general analysis that is presented in appendix A.

More importantly, using analytic and numerical results, we prove in section 3.3 that the qualitative features do not depend on $\nu$ when its value is finite. However, there are drastic changes when comparing with the case $\nu=+\infty$ studied in [1], e.g. the existence of critical points. We will discuss in great detail all these properties in section 4 for the particular case $\nu=3$.

\subsection{Local thermodynamic stability conditions}

Local thermodynamic stability follows from studying the heat capacity and electric permittivity,

$$
C_{Q} \equiv T\left(\frac{\partial S}{\partial T}\right)_{Q}, \quad \epsilon_{T} \equiv\left(\frac{\partial Q}{\partial \Phi}\right)_{T}
$$

Concretely, these response functions should be positively defined [47]. By imposing that the energy is a minimum at the thermodynamic equilibrium (or, equivalently, that the entropy is a maximum), and by performing small fluctuations in $T$ and $Q$ around that configuration, it follows that the local stability conditions are:

$$
\begin{array}{r}
\left(\frac{\partial^{2} M}{\partial S^{2}}\right)_{Q}=T C_{Q}^{-1} \geq 0 \quad \rightarrow \quad C_{Q} \geq 0 \\
\left(\frac{\partial^{2} M}{\partial Q^{2}}\right)_{S}=\epsilon_{S}^{-1} \geq 0 \quad \rightarrow \quad \epsilon_{S} \geq 0 \\
\left(\frac{\partial^{2} M}{\partial Q^{2}}\right)_{S}\left(\frac{\partial^{2} M}{\partial S^{2}}\right)_{Q}-\left[\left(\frac{\partial}{\partial S}\right)_{Q}\left(\frac{\partial M}{\partial Q}\right)_{S}\right]^{2}=T C_{Q}^{-1}\left(\epsilon_{S}^{-1}-T C_{Q}^{-1} \alpha_{Q}^{2}\right) \geq 0,
\end{array}
$$

where $\alpha_{Q}:=(\partial \Phi / \partial T)_{Q}$. The last condition is associated to the physical situation when both fluctuations are turned on simultaneously. We can use the well known thermodynamic relations

$$
C_{\Phi}=C_{Q}+T \epsilon_{T} \alpha_{Q}^{2}, \quad \epsilon_{S}=\epsilon_{T}-\frac{T \alpha_{\Phi}^{2}}{C_{\Phi}}, \quad \alpha_{\Phi}=-\epsilon_{T} \alpha_{Q}
$$

where $\alpha_{\Phi}:=(\partial Q / \partial T)_{\Phi}$, to show that the three conditions (3.2), (3.3), and (3.4) are equivalent to

$$
C_{Q} \geq 0, \quad \epsilon_{T} \geq 0, \quad C_{Q} \epsilon_{T}\left(C_{Q}+T \epsilon_{T} \alpha_{Q}^{2}\right)^{-1} \geq 0
$$

Notice that, by using the relations (3.5), $C_{Q} \geq 0$ and $\epsilon_{T} \geq 0$ implies $C_{\Phi} \geq 0$ and $\epsilon_{S} \geq 0$.

In order to understand the local thermodynamic stability conditions in a given ensemble from a physical point of view, consider first an equilibrium configuration in the 


\begin{tabular}{|c|c|c|}
\hline Name & Definition & Motivation \\
\hline$\Phi_{0}$ & $\left(\frac{\partial T}{\partial S}\right)_{\Phi=\Phi_{0}}=\left(\frac{\partial^{2} T}{\partial S^{2}}\right)_{\Phi=\Phi_{0}}=0$ & Critical point - grand-canonical ensemble \\
\hline$\Phi^{*}$ & $\Phi\left(x_{+}=\infty\right)$ & A common end point of all the isoterms \\
\hline$Q_{0}$ & $\left(\frac{\partial T}{\partial S}\right)_{Q=Q_{0}}=\left(\frac{\partial^{2} T}{\partial S^{2}}\right)_{Q=Q_{0}}=0$ & Critical point - canonical ensemble \\
\hline$Q^{*}$ & $Q\left(x_{+}=\infty\right)$ & A common end point of all the isotherms \\
\hline$T_{0}$ & $\left(\frac{\partial Q}{\partial \Phi}\right)_{T=T_{0}}=\left(\frac{\partial^{2} Q}{\partial \Phi^{2}}\right)_{T=T_{0}}=0$ & Critical point - canonical ensemble \\
\hline$T_{\infty}$ & $\left(\frac{\partial \Phi}{\partial Q}\right)_{T=T_{\infty}}=\left(\frac{\partial^{2} \Phi}{\partial Q^{2}}\right)_{T=T_{\infty}}=0$ & Critical point - grand-canonical ensemble \\
\hline
\end{tabular}

Table 1. Definition of the set of variables used to characterize distinct features in the plots of equation of state for each ensemble.

canonical ensemble, where both the electric charge $Q$ and Hawking temperature $T$ are kept fixed. The only quantities we can freely vary are the mass of the black hole and the conjugate potential. Since the charge is fixed, the conjugate potential is a function of mass, $\Phi=\Phi(M)$, and so a variation of the conjugate potential comes as a consequence of variations of the mass, which, in turn, defines the sign of $C_{Q}$. Thus, in the canonical ensemble, the only requirement for local thermodynamic stability is $C_{Q} \geq 0 .{ }^{6}$ In the grand-canonical ensemble, there is more freedom since a variation of the entropy could come from variations of the mass and the electric charge.

\subsection{The general criterion}

In this section, we introduce a criterion that can be used to analyze the local stability for any finite value of the parameter $\nu$. It consists of carrying out a careful graphical analysis of the equation of state and the phase diagrams. In order to facilitate the analysis, it is convenient to introduce a set of useful variables, which are presented in table 1 . For the sake of simplicity, we are going to work with the dimensionless version of the variables, given by

$$
\eta \rightarrow \sqrt{\alpha} \eta, \quad M \rightarrow \sqrt{\alpha} M, \quad T \rightarrow \frac{T}{\sqrt{\alpha}}, \quad S \rightarrow \alpha S, \quad Q \rightarrow \sqrt{\alpha} Q
$$

so that $\alpha$ is not going to appear explicitly in the relevant physical expressions.

The general analysis to be performed for arbitrary values of $\nu$ in the potential consists basically of the following two steps:

1) We analize the diagrams $Q-\Phi-T$ and $S-T-Q$ or $S-T-\Phi$ (depending on the ensemble) and identify the critical curves where the behaviour changes;

\footnotetext{
${ }^{6}$ This condition is widely used in the literature. However, we would like to point out a subtlety that is not considered when studying black holes in a box. In this specific case, in principle, one can vary independently the chemical potential by moving the walls of the box and so one could also consider the permittivity as a response function.
} 
2) We translate the relevant points, curves, and regions from one diagram to another. Since the slopes in those diagrams are directly related with the sign of the response functions, we are able to determine in what regions they are simultaneously positive.

In order to facilitate the thermodynamic analysis, it is worth showing that the equation of state for each finite value of the parameter $\nu$ behaves in a similar fashion, so that we can draw general conclusions regarding the thermodynamic stability of a black hole by just studying a particular case. We are going to show that the general behaviour of a black hole configuration is independent of the parameter $\nu$ once we have (i) the asymptotic value for each thermodynamic quantity and (ii) the existence or not of critical points.

\subsection{Universal features for any finite value of $\nu$}

Since a detailed study to delimit the regions of stability is quite cumbersome, we leave the application of the stability criterion, presented above, to the general case for appendix A. In this section, instead, we focus on the universality of thermodynamic behaviour for any finite value of the parameter $\nu$. We use a combination of numerical and analytic methods to find the critical points and curves that are relevant for the stability regions.

\subsubsection{Grand-canonical ensemble, $T$ and $\Phi$ fixed}

Due to the stability conditions outlined in the previous section, we only have to study $Q$ vs $\Phi$ at fixed entropy and $S$ vs $T$ at fixed conjugate potential. By using the expressions for the thermodynamic quantities, we can express the electric charge and the conjugate potential as

$$
Q=\frac{1}{4 \pi} \sqrt{\frac{2 S\left(x_{+}^{\nu}-1\right)(\nu-1) A_{S}}{\left(\nu^{2}-4\right) \nu^{3}}}, \quad \Phi=\sqrt{\frac{2(\nu-1)\left(x_{+}^{\nu}-1\right) A_{S}}{\pi \nu^{3}\left(\nu^{2}-4\right) x_{+}^{1+\nu}}}
$$

where

$$
A_{S} \equiv\left[(\nu-2) x_{+}^{\nu+2}-\left(\nu^{2}-4\right) x_{+}^{2}-(\nu+2) x_{+}^{2-\nu}+\nu^{2}\right] S+\pi \nu^{2} x_{+}\left(\nu^{2}-4\right) \text {. }
$$

Since the entropy is constant, one can check that there are no inflection points and $Q$ is a monotonically increasing function of $\Phi$. This means that the only possible type of curves in the $Q$ vs $\Phi$ diagram for a fixed value of the entropy starts at the origin and have positive definite slopes. We emphasize that this behaviour is independent of $\nu$.

Let us now explore the general features of the $S$ vs $T$ diagram with fixed conjugate potential. We have

$$
S=\frac{\pi \nu^{2} x_{+}^{\nu-1}\left(\nu^{2}-4\right) A_{\Phi}}{2(\nu-1)\left(x_{+}^{\nu}-1\right) E_{\Phi}}, \quad T= \pm \frac{\sqrt{2}\left[2(\nu-1)\left(x_{+}^{\nu}-1\right) B_{\Phi}-\Phi^{2} C_{\Phi}\right] x_{+}^{1-\nu}}{8 \pi \sqrt{A_{\Phi}(\nu-1)\left(x_{+}^{\nu}-1\right)\left(\nu^{2}-4\right) E_{\Phi}}}
$$


where the plus sing in front of the expression for the temperature is for $1<\nu<2$ and the negative one for $\nu>2$. To write the equations (3.10) in a compact form, we have defined

$$
\begin{aligned}
& A_{\Phi} \equiv 2\left(x_{+}^{\nu}-1\right)(\nu-1) x_{+}^{2-\nu}-\Phi^{2} \nu x_{+}^{2}, \\
& B_{\Phi} \equiv\left(\nu x_{+}^{2}-2 x_{+}^{2}-\nu-2\right) x_{+}^{\nu}+\nu x_{+}^{2}+2 x_{+}^{2}-\nu+2, \\
& C_{\Phi} \equiv\left[x_{+}^{2}\left(\nu^{2}-3 \nu+2\right)-\nu(\nu+2)\right] x_{+}^{2 \nu}+2\left[x_{+}^{2}(\nu+2)-\nu\right](\nu-1) x_{+}^{\nu}+(\nu+2) x_{+}^{2}, \\
& E_{\Phi} \equiv(\nu+2) x_{+}^{2-\nu}+(2-\nu) x_{+}^{\nu+2}+\left(x_{+}^{2}-1\right) \nu^{2}-4 x_{+}^{2}
\end{aligned}
$$

We can check the sign of temperature in the limits $x_{+}=1$ and $x_{+} \rightarrow \infty$ (corresponding to the boundary and singularity, respectively)

$$
\lim _{x_{+} \rightarrow 1} T=0, \quad \lim _{x_{+} \rightarrow \infty} T=\left\{\begin{array}{rl}
-\infty & \text { if } \Phi>\Phi^{*} \\
0 & \text { if } \Phi=\Phi^{*} \\
+\infty & \text { if } \Phi<\Phi^{*}
\end{array},\right.
$$

where $\Phi^{*} \equiv \sqrt{2}$. Notice that the limits for the temperature (and also for the entropy) are independent of the value of $\nu$.

There are then two different situations, when the value of the conjugate potential exceeds or it is inferior to $\Phi^{*}$. In the first case, $\Phi>\Phi^{*}$, we observe that by increasing the horizon coordinate, $x_{+}$, the temperature goes to $-\infty$. However, one can check that this can be done through a range of positive values, when there exists a maximum positive value of the temperature, for certain values of $\Phi$. That is, regular black hole solutions can be obtained in the range $\Phi \in[\sqrt{2}, 2)$.

In terms of the coordinate $x$ that is related to the normal radial coordinate in flat space by $x \simeq 1+1 /(\eta r)$, we emphasize that there exists another value, $x=x_{+}^{\max }$, for which the temperature vanishes. Therefore, for the black holes characterized by $\Phi \in[\sqrt{2}, 2)$, the horizon coordinate ranges as $1<x_{+}<x_{+}^{\max }<\infty$, where $T\left(x_{+}^{\max }\right)=0$, so that $S \geq S_{\min } \equiv S\left(x_{+}^{\max }\right)$. These results can be graphically understood and they are presented in section 4 for the particular value $\nu=3$.

Let us consider now the case $\Phi<\Phi^{*}$. There exists a critical point, whose temperature $T_{\infty}$ is defined in table 1 . Indeed, this specific critical point can be numerically obtained for a given value of $\nu$ and the result is presented in figure 1a. Interestingly, the critical point is located on the critical line $\Phi_{0}=\Phi_{0}(\nu)$ whose value is slightly lower than $\Phi^{*}$ (see figure $1 \mathrm{~b}$ ). The existence of $T_{\infty}$ for each value of $\nu$ implies that this critical behaviour is a generic property of the black hole solution under consideration.

\subsubsection{Canonical ensemble, $T$ and $Q$ fixed}

In the canonical ensemble, the relevant diagrams are $Q$ vs $\Phi$ at fixed $T$ and $S$ vs $T$ with fixed electric charge. The electric charge and its conjugate potential can be expressed in terms of $x_{+}$and $T$ in a parametric way, by

$$
Q=\frac{\Phi \nu\left(\nu^{2}-4\right) x^{\nu+1}}{4\left|C_{T}\right|}, \quad \Phi=\frac{\sqrt{8 \pi T\left(C_{T}+B_{T}\right)\left(x_{+}^{\nu}-1\right)\left(\nu^{2}-4\right)(\nu-1)}}{\left|C_{T}\right|}
$$




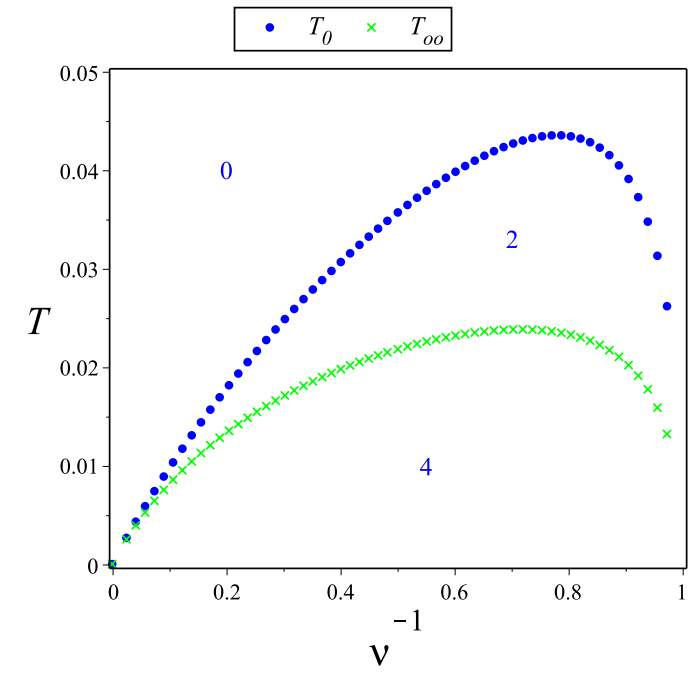

(a) Critical temperatures $T_{0}$ and $T_{\infty}$ as functions of $\nu$

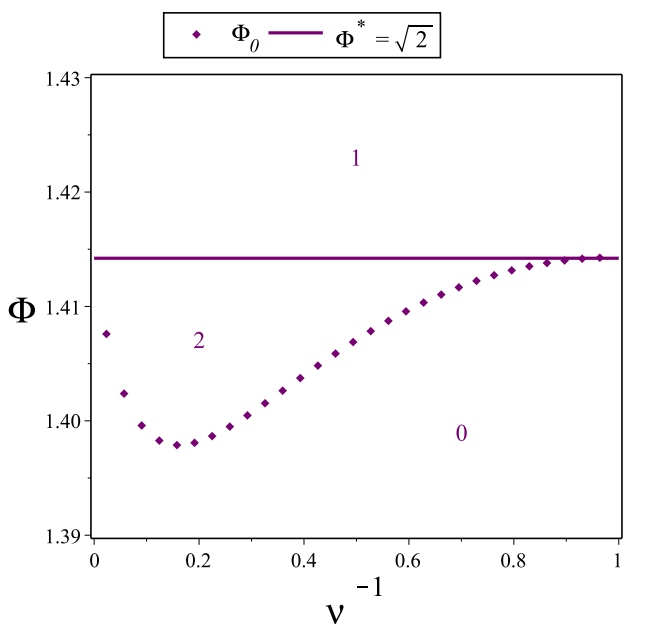

(b) Critical conjugate potentials

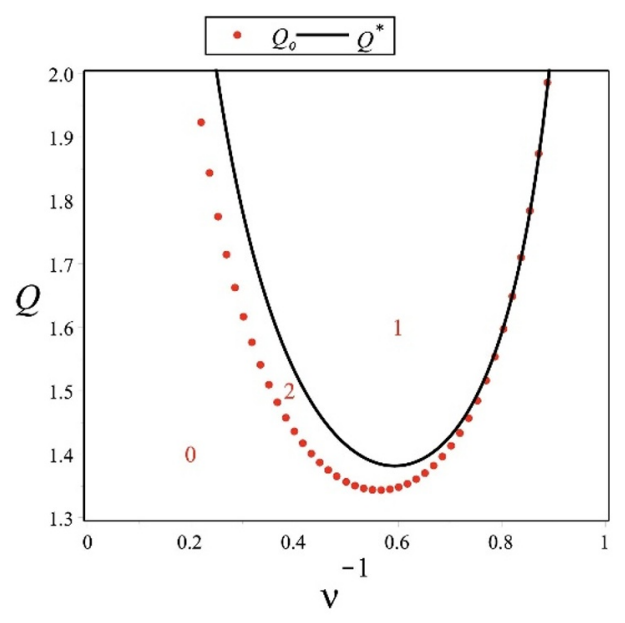

(c) Critical electric charges

Figure 1. Each number that appears on the plots indicates the maximum number of times that $\epsilon_{T}, C_{Q}$, and $C_{\Phi}$ change their signs, respectively. This is used in the analysis performed in appendix A. The plots show: (a) critical temperatures $T_{0}=T_{0}(\nu)$ and $T_{\infty}=T_{\infty}(\nu)$. Note that $T_{0}>T_{\infty}$, with the equality holding in the limit $\nu=1$ and $\nu \rightarrow \infty$; (b) critical electric charges $Q_{0}=Q_{0}(\nu)$ and $Q^{*}=Q^{*}(\nu)$. Note that, in general, $Q_{0}<Q^{*}$; (c) critical conjugate potentials $\Phi_{0}=\Phi_{0}(\nu)$ and $\Phi^{*}(=\sqrt{2})$. Note that, in general, $\Phi_{0}<\Phi^{*}$.

where we have defined

$$
\begin{aligned}
A_{T} \equiv & {\left[4 \pi^{2} \nu^{2}\left(\nu^{2}-4\right) T^{2}+x_{+}^{2}(\nu-1)(\nu-2)-\nu(\nu+1)\right] x_{+}^{2 \nu}+(\nu+2) x_{+}^{2}+} \\
& 2\left[\left(x_{+}^{2}-1\right) \nu+2 x_{+}^{2}\right](\nu-1) x_{+}^{\nu}, \\
B_{T} \equiv & \left(x_{+}^{2}-x_{+}^{\nu}\right)(\nu+2)+\left(x_{+}^{\nu+2}-1\right)(\nu-2), \\
C_{T} \equiv & 2 \pi \nu x_{+}^{\nu}\left(\nu^{2}-4\right) T \mp \sqrt{\left(\nu^{2}-4\right) A_{T}\left(x_{+}, T\right)} .
\end{aligned}
$$


The $\mp$ signs in the equations (3.16) correspond to the interval $1<\nu<2$ (the upper sign) and $\nu>2$ (the lower sign), respectively. For the isotherm $T=0$, we have the following limits,

$$
\begin{array}{lrl}
\lim _{x_{+} \rightarrow \infty} \Phi(T=0) \equiv \Phi^{*}=\sqrt{2}, & \lim _{x_{+} \rightarrow 1^{+}} \Phi(T=0)=2, \\
\lim _{x_{+} \rightarrow \infty} Q(T=0) \equiv Q^{*}=\frac{\nu \sqrt{2} \sqrt{\nu+2}}{4 \sqrt{\nu-1}}, & \lim _{x_{+} \rightarrow 1^{+}} Q(T=0)=\infty
\end{array}
$$

from where we note that the isotherm $T=0$ has one ending point at $\left(Q^{*}, \Phi^{*}\right)$. We emphasize that, in fact, the point $\left(Q^{*}, \Phi^{*}\right)$ does not depend of the temperature, but it is more convenient to compute these values taking first the limit $T=0$. Unlike the extremal $\mathrm{RN}$ black hole for which the conjugate potential is constant, in the extremal limit of the hairy black hole, even if there is a constrain between the mass and charge, the conjugate potential is not fixed and it still depends of one independent conserved charge. Therefore, the point $\left(Q^{*}, \Phi^{*}\right)$ exists for any $T \geq 0$ and the main observation is that it is the endpoint of all isotherms (the other endpoint, for $T>0$, is, as expected, $(Q, \Phi)=(0,0))$. The limit $\nu \rightarrow+\infty$ can not be taken directly in the expression of $Q^{*}$, but it was shown in [1] that a similar endpoint of all isotherms exists and its values are $\left(Q^{*}, \Phi^{*}\right)=(0, \sqrt{2})$. Despite the dependence of $Q^{*}$ on the value of $\nu$, its existence is a general feature independent of $\nu$.

Interestingly, it can be shown that for any finite value of $\nu$, the equation of state, $\Phi=\Phi(Q, T)$, contains two critical isotherms, each one coming with its own critical point. With the notations from in table 1 , the first one corresponds to $T_{0}$ and the second one to $T_{\infty}$. Both of them are depicted, as a function of $\nu$, in figure $1 \mathbf{a}$ and we point out that $T_{0}>T_{\infty}$.

Let us now investigate the general features of the $S$ vs $T$ diagram at fixed $Q$. We have

$$
S=\frac{16 \pi \nu^{2} Q^{2}\left(\nu^{2}-4\right)\left(x_{+}^{\nu}-1\right)}{B_{Q}}, \quad T= \pm \frac{16\left(x_{+}^{\nu}-1\right) Q^{2} A_{Q}-x_{+}^{1+\nu} B_{Q}}{16 \nu \pi Q \sqrt{x_{+}^{3 \nu+1}\left(\nu^{2}-4\right)\left(x_{+}^{\nu}-1\right) B_{Q}}}
$$

where we have defined

$$
\begin{aligned}
A_{Q} \equiv & {\left[(\nu-2) x_{+}^{2 \nu+2}-2 \nu x_{+}^{\nu}\right](\nu-1)+\left[2(\nu-1) x_{+}^{\nu+2}+x_{+}^{2}-\nu x_{+}^{2 \nu}\right](\nu+2), } \\
B_{Q} \equiv & \nu x_{+}\left(x_{+}^{\nu}-1\right)^{2}(\nu-1)\left(\nu^{2}-4\right) \mp \sqrt{x_{+}^{-\nu} \nu(\nu-1)\left(\nu^{2}-4\right)\left(x_{+}^{\nu}-1\right)^{3} E_{Q},} \\
E_{Q} \equiv & 32\left[(\nu-2) x_{+}^{2 \nu+2}-\left(\nu^{2}-4\right) x_{+}^{\nu+2}+\nu^{2} x_{+}^{\nu}-(\nu+2) x_{+}^{2}\right] Q^{2}+ \\
& x_{+}^{\nu+2} \nu(\nu-1)\left(\nu^{2}-4\right)\left(x_{+}^{\nu}-1\right)
\end{aligned}
$$

The minus sign in the equations above corresponds to the interval $1<\nu<2$ and the plus sign is used for $\nu>2$. The limits of interest are now

$$
\lim _{x_{+} \rightarrow 1^{+}} T=0, \quad \lim _{x_{+} \rightarrow \infty} T=\left\{\begin{array}{rl}
-\infty & \text { if } Q>Q^{*} \\
0 & \text { if } Q=Q^{*} \\
+\infty & \text { if } Q<Q^{*}
\end{array} .\right.
$$


Notice that these limits are similar with the ones for $\Phi$ fixed (3.15), but now in canonical ensemble at fixed charge, therefore a similar analysis can be done. One important difference is that there exist regular black holes for any $Q>Q^{*}$. However, when $Q<Q^{*}$ there exists a critical point that can be numerically obtained for a given value of $\nu$. Such a point is located on the critical line $Q=Q_{0}(\nu)$ and it occurs at the temperature $T=T_{0}(\nu)$. The quantities $Q^{*}, Q_{0}$, and $T_{0}$ are plotted as functions of $\nu$ in figure $1 \mathrm{a}$ and figure $1 \mathrm{c}$ and, as long as $\nu$ is finite, $T_{0}>T_{\infty}, Q_{0}<Q^{*}$, and $\Phi_{0}<\Phi^{*}$.

We would like to emphasize that the critical behaviour is a feature of these solutions for any finite value of $\nu$. In the $\nu=\infty$ limit, there is no critical behaviour [1]. Since the critical quantities exist for any value of $\nu$, in the next section we will analyze the local stability of the particular solution $\nu=3$. Nevertheless, the general study of stability regions made for a generic $\nu$ can be found in appendix A.

\section{Comparison between hairy solutions with $\nu=3$ and $\nu=\infty$}

In this section, we perform a detailed analysis of the local stability for a specific solution corresponding to the value $\nu=3$ in the equations (2.5)-(2.10). We concretely identify the new features pointed out in the previous section and compare these results with the ones obtained previously in [1] for the particular limit $\nu \rightarrow \infty$. Interestingly, when $\nu$ has finite values, there exist critical points in both, canonical and grand-canonical, ensembles and, also, there exists a new branch of stable black holes.

\subsection{Hairy black hole solution with $\nu=3$}

The self-interaction of the scalar field in this case is governed by the following potential:

$$
V(\phi)=\frac{4 \alpha}{45}\left[\sinh (\sqrt{2} \phi)-2 \sinh \left(\frac{\phi}{\sqrt{2}}\right)+16 \sinh \left(\frac{\phi}{8}\right)\right]
$$

The metric functions are

$$
\Omega(x)=\frac{9 x^{2}}{\eta^{2}\left(x^{3}-1\right)^{2}}, \quad f(x)=\frac{\alpha\left(x^{6}-5 x^{3}+9 x-5\right)}{45 x}+\frac{\eta^{2}\left(x^{3}-1\right)^{2}}{9 x}\left[1-\frac{q^{2}\left(x^{3}-1\right)}{12 x^{3}}\right]
$$

We can expand the component of the metric in canonical coordinates by solving $\Omega(x) \approx r^{2}$ in the asymptotic region, where $r$ is the radial coordinate. That is

$$
x=\frac{2^{1 / 3}\left[(\eta r)^{3 / 2}+\sqrt{(\eta r)^{3}-4}\right]^{2 / 3}+2}{2^{2 / 3} \sqrt{\eta r}\left[(\eta r)^{3 / 2}+\sqrt{(\eta r)^{3}-4}\right]^{1 / 3}}=1+\frac{1}{\eta r}-\frac{1}{3(\eta r)^{3}}+\frac{1}{3(\eta r)^{4}}+\mathcal{O}\left(r^{-6}\right)
$$

chosen consistently with the existence domain of the positive branch, $x>1$ for $r>0 .^{7}$ Without the loss of generality, we work with positive values for the parameter $\eta$. The boundary of spacetime located at $x=1$ corresponds to $r \rightarrow \infty$. In flat spacetime, in

\footnotetext{
${ }^{7}$ We emphasize that the general solution has also a 'negative branch' with the domain $0 \leq x<1$ that we do not consider in this work.
} 
general, the scalar field has the fall-off $\phi=\phi_{\infty}+\frac{\Sigma}{r}+\mathcal{O}\left(r^{-2}\right)$. However, due to the presence of the potential $V(\phi)$, the asymptotic value of the scalar field is fixed to $\phi_{\infty}=0$. Therefore, the scalar field, $\phi(x)=2 \sqrt{2} \ln (x)$, has the following asymptotic form

$$
\phi(r)=\frac{2 \sqrt{2}}{\eta r}-\frac{\sqrt{2}}{\eta^{2} r^{2}}+\mathcal{O}\left(r^{-3}\right)
$$

The fisrt term in the expansion (4.4) provides the scalar charge [48] $\Sigma$ that, in fact, is not an independent parameter of the solution, ${ }^{8}$ but is determined by the conserved charges, namely, $M$ and $Q$, by the relation

$$
M=\frac{4 \sqrt{2} Q^{2}}{\Sigma}-\frac{\sqrt{2} \Sigma\left(\alpha \Sigma^{2}+24\right)}{192}
$$

This is consistent with what we have claimed before, namely that the scalar field is 'secondary hair' and its degrees of freedom live outside the horizon.

\subsection{Equation of state and critical points}

Since we have rescaled all the relevant dimensionful quantities according to (3.7), in what follows the thermodynamic variables are going to appear dimensionless. The study of the equation of state $\Phi=\Phi(Q, T)$ is important for determining local stability. Since it is not possible to explicitly solve for $x_{+}$from the equations (3.16), we perform a graphical analysis of the equations in parametric form. The equation of state for $\nu=3$ is plotted in figure 2 where it can be observed that all the isotherm curves end on the line $\Phi^{*}=\sqrt{2}$, $Q^{*}=3 \sqrt{5} / 4 \approx 1.6771$, consistently with the corresponding limits in (3.20) and (3.21). One important difference between the thermodynamic behaviour of hairy black hole solutions with finite $\nu$ and $\nu=\infty$ is that, for the solution $\nu=\infty$, the charge is $Q^{*}=0$ while its conjugate potential is still $\Phi^{*}=\sqrt{2} .{ }^{9}$ The plot in the right-hand side of figure 2 shows in more detail the fold with two critical isotherms. These two critical isotherms are determined by the equations presented in table 1 . For $\nu=3$, the critical temperatures are $T_{0} \approx 0.02675$ and $T_{\infty} \approx 0.01803$.

By selecting an arbitrary isotherm, we can distinguish various branches where the response function $\epsilon_{T}$ changes its sign. If $T>T_{0}$, for instance, $\epsilon_{T}$ becomes positive definite, but, as we will see next, the heat capacity $C_{Q}$ becomes negative definite and hence there are no thermodynamically stable black holes. The most interesting case occurs for 'low' temperature regimes, $T<T_{0}$ and, more restrictively, $T<T_{\infty}$.

In figure 3 , we consider various isotherm curves and, in the right hand side panel, we identify the two critical isotherms mentioned in the previous section for a general $\nu$. The critical points are located at $\left(\Phi\left(Q_{0}, T_{0}\right), Q_{0}\right)$ and at $\left(\Phi_{0}, Q\left(\Phi_{0}, T_{\infty}\right)\right)$, respectively. Let us consider an isotherm $0<T<T_{\infty}$ (for instance, $T=0.005$ from the first panel in figure 3 ). By starting from the configuration at $Q=0$ and $\Phi=0$, that is, from the limit $x=\infty$, we

\footnotetext{
${ }^{8}$ For discussions on the scalar charges and their role on the first law of thermodynamics, see [45, 49, 50].

${ }^{9}$ Notice that $Q^{*}=0$ for the limit $\nu=\infty$ does not follows from (3.21). The solution $\nu=\infty$ is actually obtained by doing a special rescaling, see [8]. Also, since we use a different convention for the action, there is an $1 / \sqrt{2}$ extra factor for the conjugate potential in [8].
} 

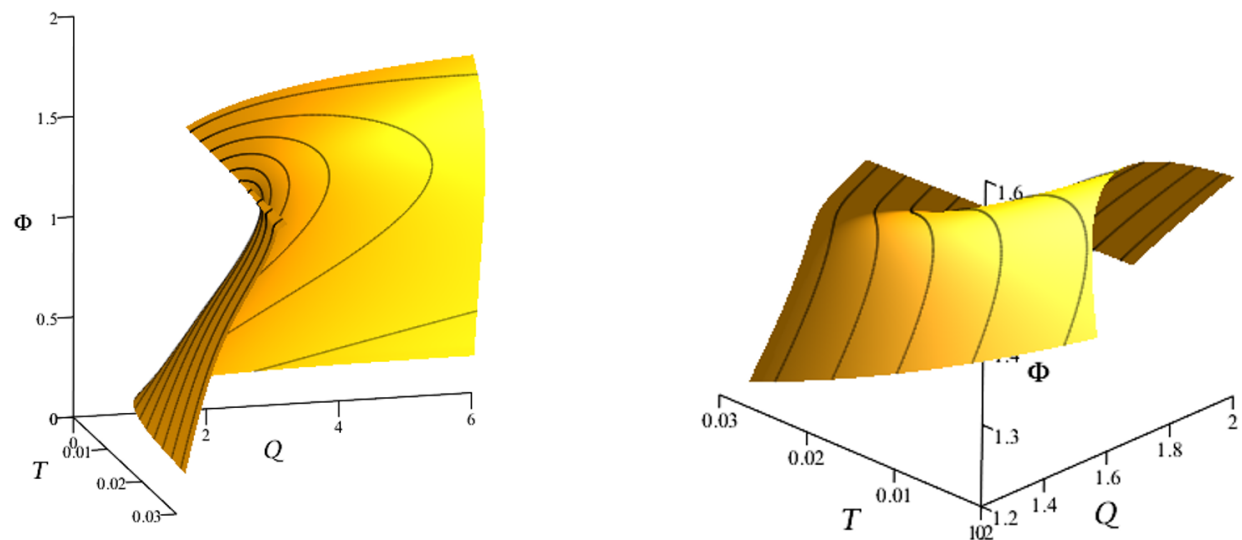

Figure 2. Left and right panels represent the equation of state (3D) for $\nu=3$ from different angles and using a different zoom. In the panel at the left hand side, we can observe the line given by the intersection of $\Phi=\Phi^{*}$ and $Q=Q^{*}$ where the isotherms stop. In the panel at the right hand side, we see 'from below' the region where the surface develops a particular fold (near $Q^{*}$ and $\Phi^{*}$ ) that is not present in the $\nu=\infty$ solution. This region contains two novel critical isotherms, $T_{\infty}$ and $T_{0}$.
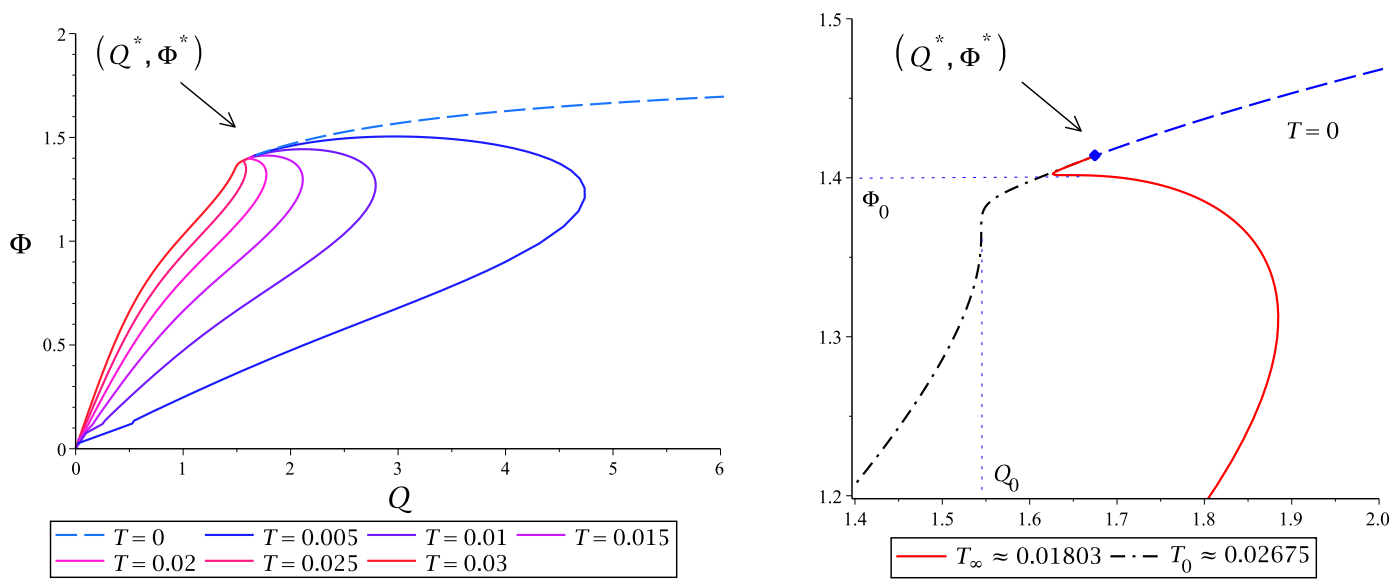

Figure 3. Left and right panels represent the equation of state (2D) for $\nu=3$. In the panel at the right hand side, three important isotherms have been chosen: $T=0$ and the two critical isotherms $T_{\infty}$ and $T_{0}$ (see the second plot in figure 2).

notice that $\epsilon_{T}>0$ until $Q$ reaches its maximum value for which $\epsilon_{T}=0$. Along this first branch, as we will see next, the heat capacity $C_{Q}$ is negative. At this point both $\epsilon_{T}$ and $C_{Q}$ change their signs. By continuing moving along this isotherm, $\epsilon_{T}<0$ until $\Phi$ reaches its maximum value, where $\epsilon_{T}$ diverges. Finally, the third branch has mostly $\epsilon_{T}>0$ and it is where stable black holes are located, since $C_{Q}>0$. 

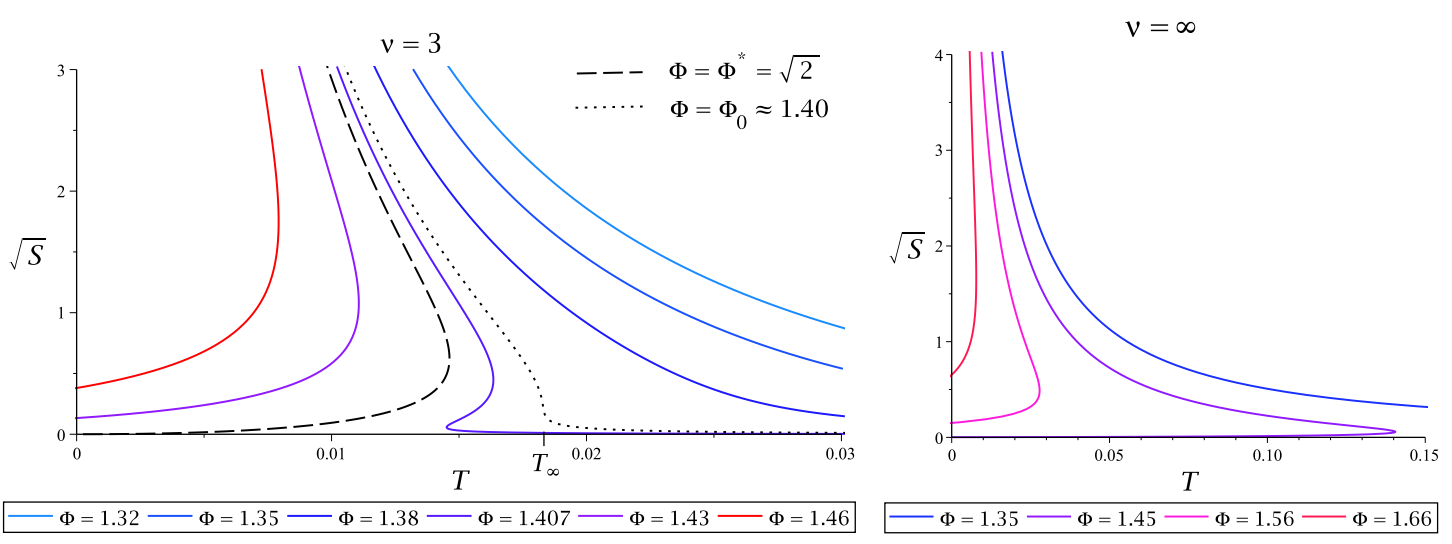

Figure 4. Phase diagram $S$ vs $T$ at fixed $\Phi$. The panel at the left hand side corresponds to the model $\nu=3$. The panel at the right hand side corresponds to the special limit $\nu=\infty$. The critical behaviour exists only for finite $\nu$. Thermally stable configurations $\left(C_{\Phi}>0\right)$ appear when $T<T_{\infty}$.

It is also important to emphasize that the isotherm $T=0$ has an horizontal asymptote at $\Phi=2$ above which there exist only naked singularities, an observation made in the general context in section 3.3.1.

\subsection{Thermodynamic stability in grand-canonical ensemble}

The criterion for the local thermodynamic stability is that heat capacity $C_{\Phi}$ and electric permittivity $\epsilon_{S}$ are simultaneously positive. However, we have determined by general arguments, in section 3.3.1, that $\epsilon_{S}>0$. This situation is basically the same as the Reissner-Nordström black hole and the special solution for $\nu=\infty$. Thus, local stability in the grand-canonical ensemble is guaranteed only by the positivity of the heat capacity $C_{\Phi}$.

The plot $S$ vs $T$ at fixed values of $\Phi$ is presented in figure 4. The panel at the left hand side corresponds to the solution $\nu=3$ and the one at the right hand side to the special limit $\nu=\infty$. One of the main difference is that, for finite $\nu$, the system exhibits a critical behaviour that is not present in the $\nu=\infty$ case. Interestingly, the novel intermediate-size black hole phase taking place within the interval $\Phi_{0}<\Phi<\Phi^{*}$ has a positive heat capacity.

Since the only non-trivial requirement for local stability in this ensemble comes from the positivity of the heat capacity $C_{\Phi}$, we can surely say that the branch of stable black holes is the one that necessarily satisfies the inequalities $\Phi_{0}<\Phi$ and $T<T_{\infty}$. Within the interval $\Phi_{0}<\Phi<\Phi^{*}$, the stable black holes are the intermediate-size black holes. We can use the equation of state to fully identify these black holes. From figure 5, we observe that if $\Phi>\Phi^{*}, x_{+}$reaches a maximum value (and so $S_{\min }$ ), as discussed in the previous section for general $\nu$. From figure 4, we observe that in this case we have two branches, the small and big black holes. These two branches, in figure 5, correspond to black holes with small and big electric charge, respectively.

A more detailed description of the critical behaviour of these solutions is going to be presented in [51]. 


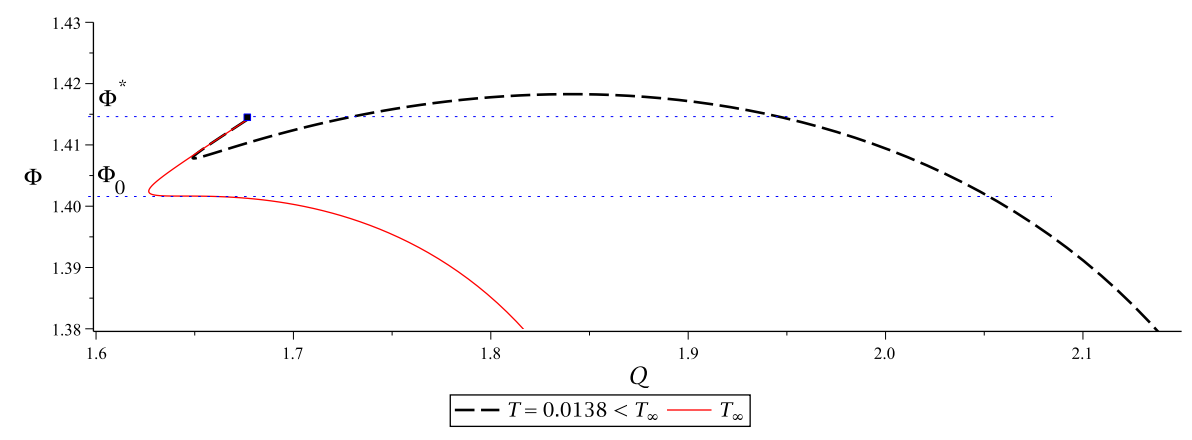

Figure 5. Stable black holes can be fully identified from the diagrams $S$ vs $T$ and $Q$ vs $\Phi$. The intersections between a fixed- $\Phi$ line and an isotherm $T<T_{\infty}$ give rise to three configurations, if $\Phi_{0}<\Phi<\Phi^{*}$, or two configurations, if $\Phi^{*}<\Phi<2$. In the first case, the configuration with small/intermediate/large value of $Q$ is also the one with the lowest/intermediate/large value of $S$. Similarly, in the second case, the configuration with the small/large value of $Q$ corresponds to the one with the small/large value of $S$ (see the first plot in figure 4).
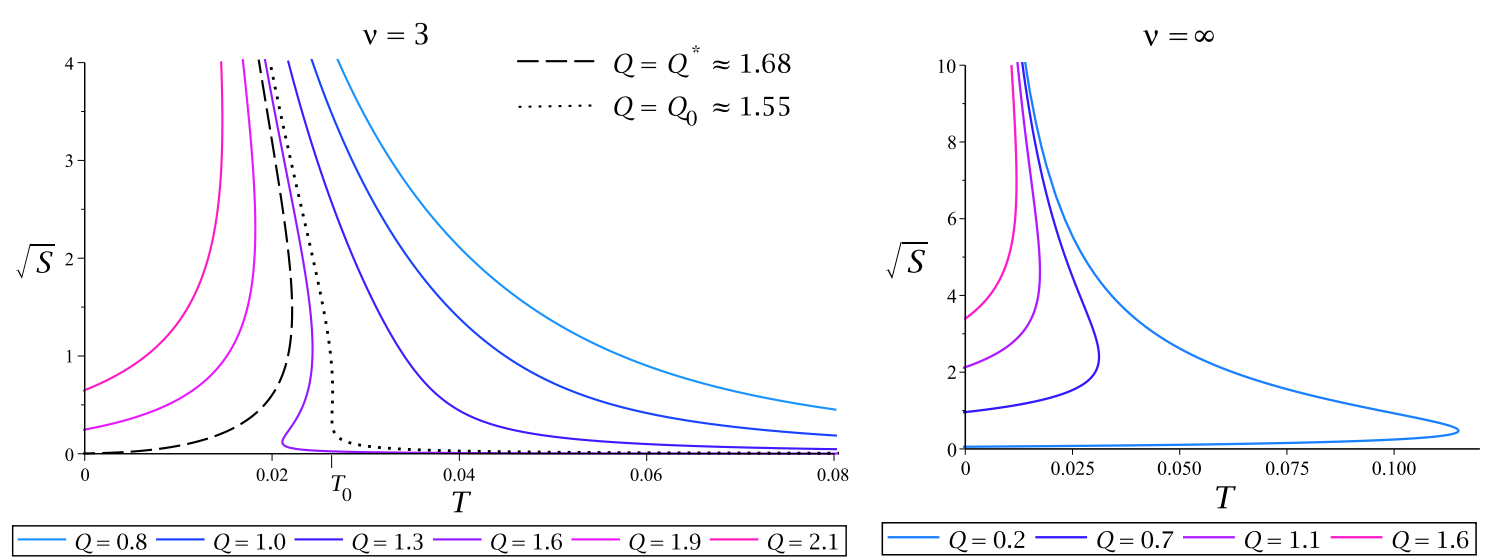

Figure 6. Phase diagram $S$ vs $T$ at fixed $Q$. The panel at the left hand side corresponds to the model $\nu=3$. The panel at the right hand side, to the special limit $\nu=\infty$. The critical behaviour exists only for finite $\nu$.

\subsection{Thermodynamic stability at $Q$ fixed}

In figure 6, we present a comparison between $S$ vs $T$ diagram at fixed $Q$ for the solution $\nu=3$ and for the special solution $\nu=\infty$. The main observation is that, unlike $\nu=\infty$ case, in this ensemble there also exist critical points. Since $T_{0}>T_{\infty}$, not all the configuration with $C_{Q}>0$ are thermodynamically stable. Concretely, there exist configurations satisfying $T_{\infty}<T<T_{0}$ that have $C_{Q}>0$, but $\epsilon_{T}<0$. To identify them, consider an isocharge line $Q_{0}<Q<Q^{*}$ and the configurations within the interval $T_{\infty}<T<T_{0}$. As we observe from figure 6 , the intermediate-size branch are stable, but, from figure 7 , we notice that the intermediate branch has a negative $\epsilon_{T}$. However, when $T<T_{\infty}$ there is a region with stable black holes. Interestingly, the intermediate-size branch with $C_{Q}>0$ in figure 6 can be in stable equilibrium as long as $0<T<T_{\infty}$ that is consistent with the analysis in section 3.3.2. 


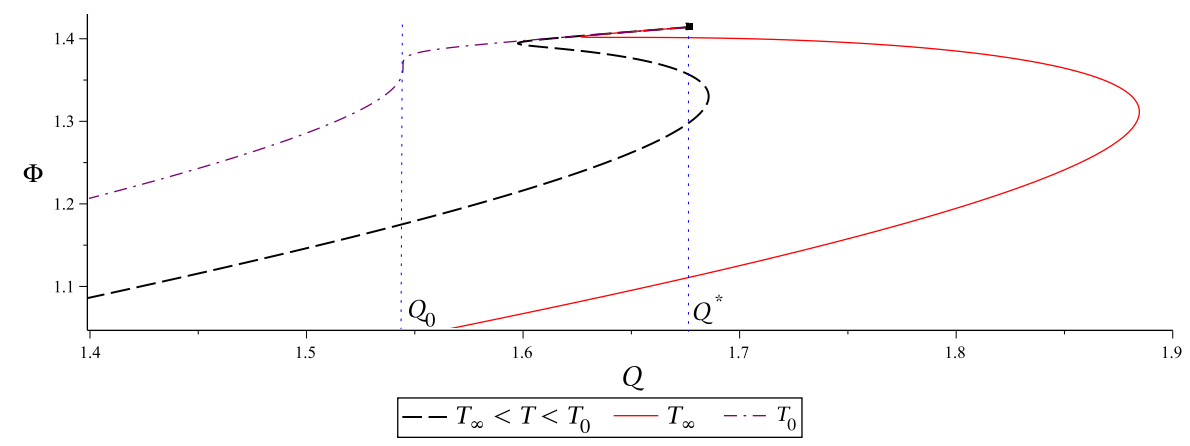

Figure 7. For the interval $T_{\infty}<T<T_{0}$, the intermediate-size branch is characterized by $C_{Q}>0$ but it has $\epsilon_{T}<0$. The analysis in the canonical ensemble confirms that stable black holes appear only for $T<T_{\infty}$.
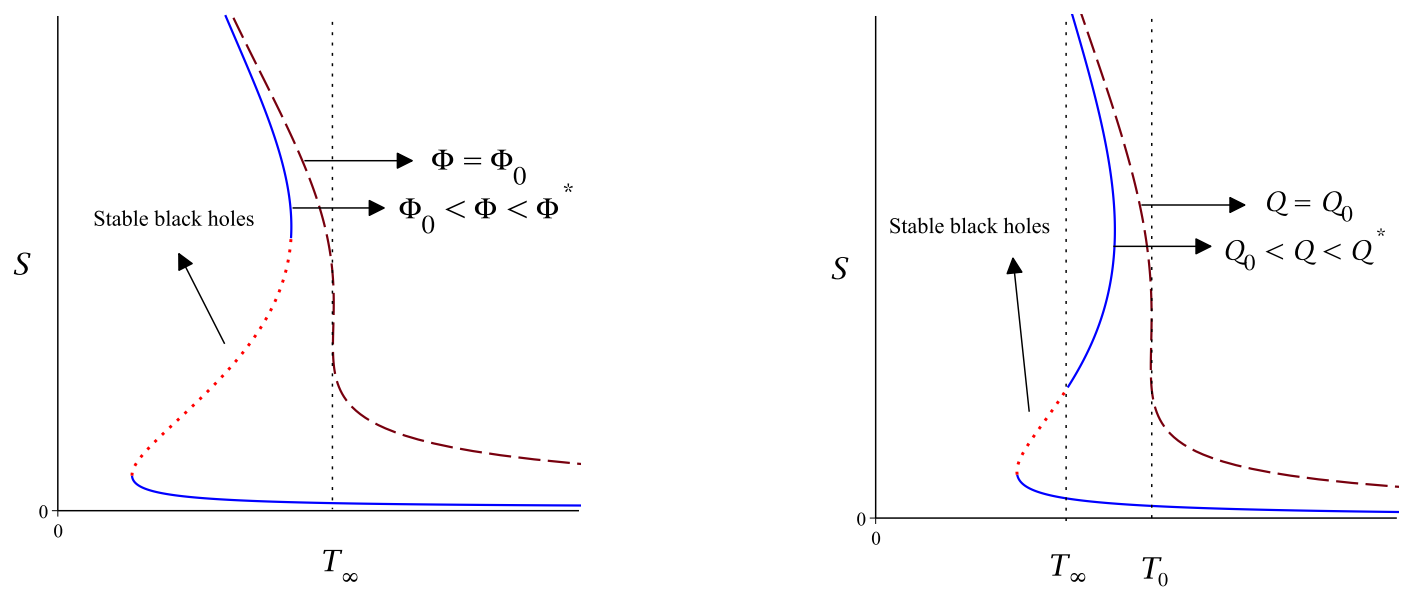

Figure 8. Intermediate-size stable black hole branches highlighted with the red dotted line, both in the grand canonical ensemble (the left hand side plot) and in the canonical one (the right hand side plot). These stable black holes, unlike the ones for $\Phi>\Phi^{*}$ and $Q>Q^{*}$ (in their respective ensembles), do not reach $T=0$ and they are characterized by only one horizon.

We are going to comment more about the new features of the hairy black hole solutions with finite $\nu$ in the 'Discussion' section.

\subsection{Extremality condition and the existence of a new branch}

Besides the existence of critical points, another interesting new feature of the hairy black holes in theories with finite $\nu$ is the appearance of a new branch of stable solutions. For concreteness, we identify this new branch in figure 8 noticing that there does not exist an extremal limit $(T \rightarrow 0)$ for these black holes.

To understand what is the origin of this new branch, we have to investigate the extremal limit. The extremality conditions, namely $f\left(x_{+}\right)=0$ and $f^{\prime}\left(x_{+}\right)=0$, can be used to get the parameters $\eta$ and $q$ as functions of $x_{+}$, and then, using the equations (2.16) and (2.18), 


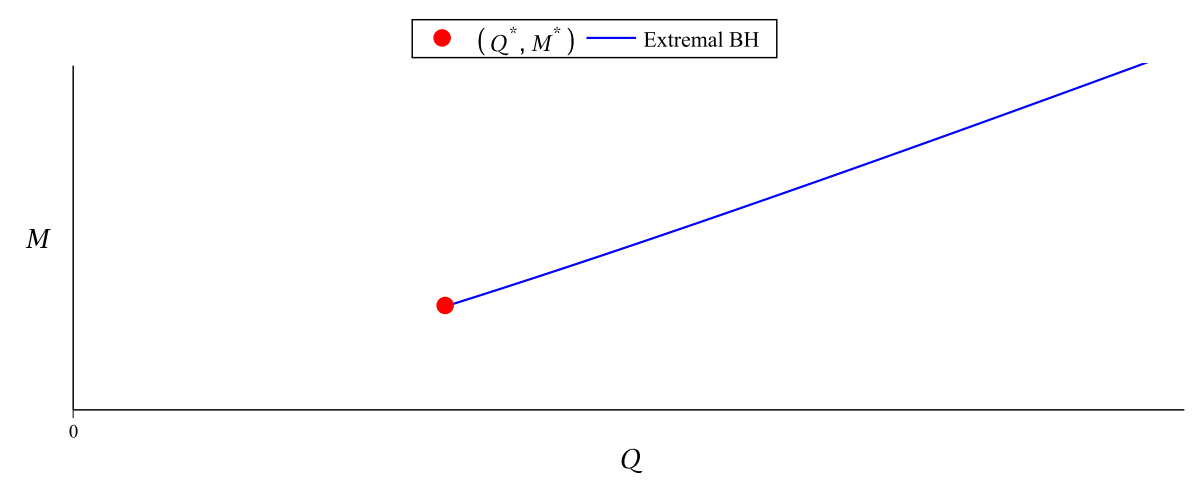

Figure 9. For black holes with $M<M^{*}$ the extremality limit does not exist.

to express $M=M\left(x_{+}\right)$and $Q=Q\left(x_{+}\right)$as

$$
\begin{aligned}
M\left(x_{+}\right) & =\frac{5}{3} \frac{8 x_{+}^{5}+32 x_{+}^{4}+35 x_{+}^{3}+28 x_{+}^{2}+22 x_{+}+10}{\left(x_{+}-1\right)^{2}\left(2 x_{+}^{2}+8 x_{+}+5\right)\left(10 x_{+}^{2}+40 x_{+}+25\right)^{\frac{1}{2}}} \\
Q\left(x_{+}\right) & =\frac{15}{2} \frac{\left(2 x_{+}^{4}+10 x_{+}^{3}+15 x_{+}^{2}+13 x_{+}+5\right)^{\frac{1}{2}}\left(x_{+}^{2}+3 x_{+}+1\right)^{\frac{1}{2}} x_{+}^{2}}{\left(x_{+}-1\right)^{2}\left(2 x_{+}^{2}+8 x_{+}+5\right)\left(10 x_{+}^{2}+40 x_{+}+25\right)^{\frac{1}{2}}}
\end{aligned}
$$

Despite the fact that these expressions are algebraically complicated and it is not possible to isolate $x_{+}$in order to obtain a simple equation for $M=M(Q)$, the plot in figure 9 is sufficient to draw some important conclusions. The relevant observation is that the extremality limit exists for black holes with masses (and charges) in a specific range. Concretely, we notice that $M$ and $Q$ are both monotonic functions of $x_{+}$and while very large black holes $\left(x_{+} \rightarrow 1\right)$ are characterized by $M \rightarrow \infty$ and $Q \rightarrow \infty$, the small ones $\left(x_{+} \rightarrow \infty\right)$ have finite minimum values $M \rightarrow M^{*}$ and $Q \rightarrow Q^{*}$, where

$$
M^{*}=\frac{2 \sqrt{10}}{3}, \quad Q^{*}=\frac{3 \sqrt{5}}{4}
$$

As a consistent check, we emphasize that $Q^{*}$ can be obtained directly from the equation (3.21) when $\nu=3$ and the extremality conditions are fixing also the mass.

Therefore, there exist regular hairy black holes with masses smaller than the one in (4.8), which do not have an extremal limit, but which are thermodynamically stable.

\section{$5 \quad$ Linear spherically symmetric perturbations}

In this section we are going to study the stability of the hairy black holes studied in the previous sections under linear spherically symmetric perturbations. The analysis follows the steps developed previously for scalarized Reissner-Nordström black holes [52-54] (see also $[9,55-62])$, and that it has also been used for black holes in alternative theories of gravity with scalar fields (see for example [63-69]). 
Up to first order in the perturbation parameter $\epsilon$, the metric (2.7) becomes

$$
d s^{2}=\Omega(x)\left[1+\epsilon e^{-i \omega t} F_{z}(x)\right]\left\{-f(x)\left[1+\epsilon e^{-i \omega t} F_{t}(x)\right] d t^{2}+\frac{\eta^{2}\left[1+\epsilon e^{-i \omega t} F_{r}(x)\right] d x^{2}}{f(x)\left[1+\epsilon e^{-i \omega t} F_{t}(x)\right]}+d \Sigma^{2}\right\}
$$

where $d \Sigma^{2} \equiv d \theta^{2}+\sin ^{2} \theta d \varphi^{2}$. Also, at first order, the perturbed electromagnetic field and scalar field are

$$
A_{t}(x, t)=a(x)\left[1+\epsilon e^{-i \omega t} F_{a}(x)\right], \quad \Phi(x, t)=\phi(x)\left[1+\epsilon e^{-i \omega t} F_{\phi}(x)\right]
$$

where $a(x)=-\frac{q}{\nu x^{\nu}}$ and $\phi(x)=\sqrt{\nu^{2}-1} \ln (x)$ are given by (2.8). The functions $F_{z}, F_{t}, F_{r}$, $F_{a}$, and $F_{\phi}$ are the perturbation functions all associated to a Fourier mode with frequency $\omega$. By choosing the gauge $F_{z}=0$, it can be shown that the equations of motion reduce to a single master equation of Schrödinger type

$$
\frac{d^{2} Z}{d R^{2}}=\left(-\omega^{2}+U\right) Z
$$

where $Z \equiv \phi \sqrt{\Omega} F_{\phi}$. The new coordinate $R$ is related to the $x$-coordinate by the transformation $d R=\frac{\eta d x}{f(x)}$ and the effective potential $U=U(x)$ is

$$
\begin{aligned}
U(x)= & {\left[\frac{2 \phi^{\prime}\left(V \gamma+V_{1}\right) \Omega^{2}}{\Omega^{\prime}}-\left(\frac{\phi^{\prime 2} f^{\prime}}{\Omega^{\prime} \eta^{2}}+\frac{4 \gamma \phi^{\prime}}{\Omega^{\prime}}-V_{2}+V \gamma^{2}\right) \Omega+\frac{2 \gamma \phi^{\prime} f^{\prime}}{\eta^{2}}-\frac{\Omega^{\prime} f^{\prime}\left(2 \gamma^{2}-1\right)}{2 \Omega \eta^{2}}+2 \gamma^{2}\right] f } \\
& +\left[\frac{\Omega^{2} \phi^{\prime 4}}{2 \eta^{2} \Omega^{\prime 2}}-\frac{\gamma \phi^{\prime 3} \Omega}{\Omega^{\prime} \eta^{2}}+\frac{\left(2 \gamma^{2}-7\right) \phi^{\prime 2}}{4 \eta^{2}}+\frac{3 \gamma \Omega^{\prime} \phi^{\prime}}{\Omega \eta^{2}}-\frac{\Omega^{\prime 2}\left(3 \gamma^{2}-1\right)}{2 \Omega^{2} \eta^{2}}\right] f^{2}
\end{aligned}
$$

where $V_{1}=\frac{d V}{d \phi}$ and $V_{2}=\frac{d^{2} V}{d \phi^{2}}$.

The stability of the solution is given by the positivity of the effective potential $U(x)$ between the event horizon and the boundary of spacetime (see for instance [70-72]). Since the domain for positive branch is $1<x<x_{+}<\infty$, it is more useful to plot $U$ vs $x^{-1}$, where $x_{+}^{-1}<x^{-1}<1$.

Near the boundary, the effective potential can be expanded as

$$
U(x)=\left[\frac{32 Q^{2} \eta^{4}}{\nu-1}+\alpha-\eta^{2}\right](x-1)^{3}+\mathcal{O}\left[(x-1)^{4}\right]
$$

and so the effective potential asymptotically vanishes. From the equation (5.4), we also observe that the effective potential vanishes at the event horizon.

A few typical profiles of the potential, as a function of $1 / x$, are presented in figure 10 . The metric function $-g_{t t}$ is represented by the dashed black line and the effective potential $U(x)$ by the solid blue line. In each panel we have plotted these functions for three distinct solutions in the theory with $\nu=3$ and $\alpha=1$. Notice that in this model, $Q^{*}=1.67705$, and we have considered $Q=2$. According to the previous analysis, when $T<0.018$, these configurations contain thermodynamically stable black holes (when $\Phi>\Phi_{0}$.). In figure 10a, we present the extremal case with $T=0$. The solution extends from the black hole horizon at $x=x_{+}$, where both the $g_{t t}$ function and the effective potential vanish, up 


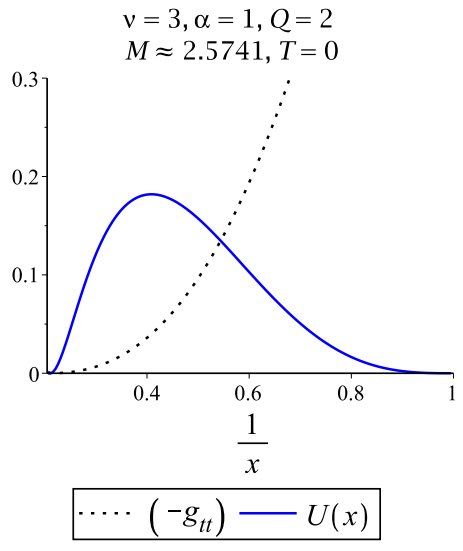

(a) $x_{+} \approx 4.7358$

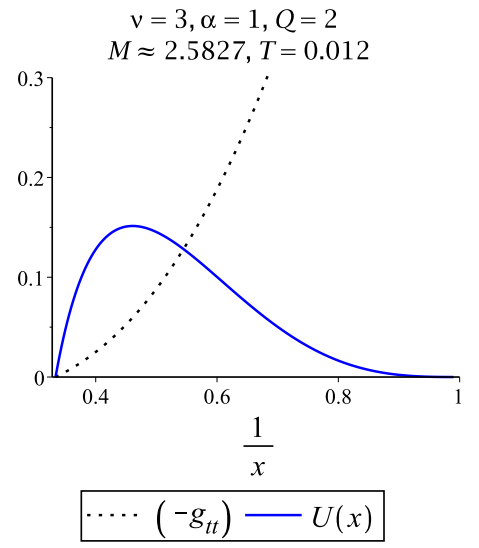

(b) $x_{+} \approx 2.9960$

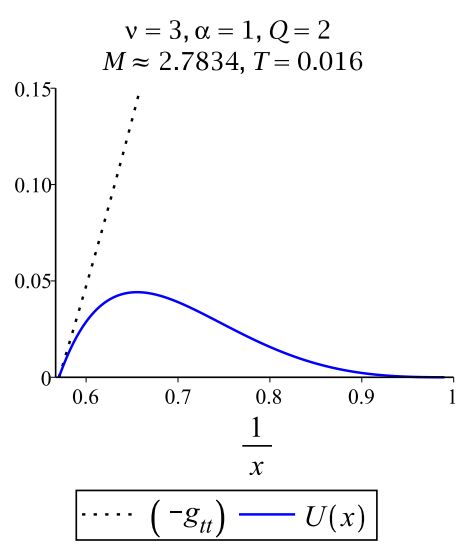

(c) $x_{+} \approx 1.7531$

Figure 10. Effective potential $U(x)$ vs $x^{-1}$ for the theory $\alpha=1$ and $\nu=3$. The electric charge of the black hole is $Q=2$. The graphics show the region between the event horizon $x=x_{+}$and the boundary $x=1$, where $-g_{t t}>0$. (a) Extremal black hole $T=0$. (b) $T=0.012$. (c) $T=0.016$.

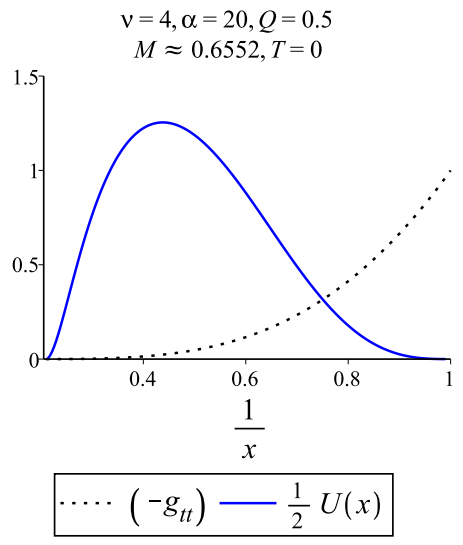

(a) $x_{+} \approx 4.7663$

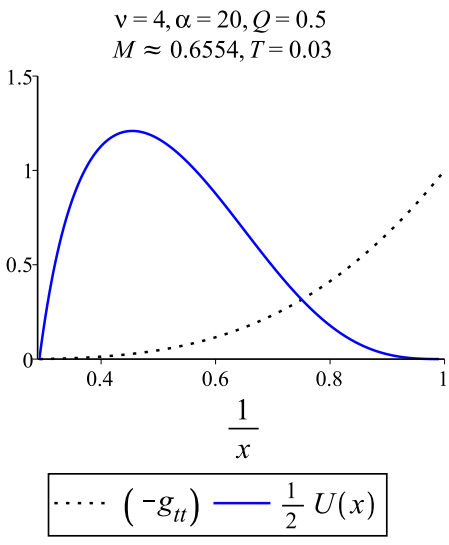

(b) $x_{+} \approx 3.4227$

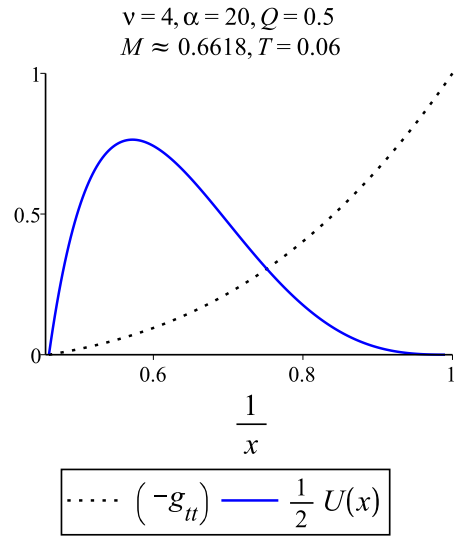

(c) $x_{+} \approx 2.1723$

Figure 11. Effective potential $U(x)$ vs $x^{-1}$ for the theory $\alpha=20$ and $\nu=4$. The electric charge of the black hole is $Q=1 / 2$. The graphics show the region between the event horizon $x=x_{+}$and the boundary $x=1$, where $-g_{t t}>0$. (a) Extremal black hole $T=0$. (b) $T=0.03$. (c) $T=0.06$.

to the asymptotic boundary at $x=1$, where $-g_{t t}=1$ and the potential vanishes again. In particular, we can see that the potential is always positive outside the horizon, meaning the solution is stable under radial perturbations. The two other solutions in figure 10b and c are non-extremal black holes with $T=0.012$ and $T=0.016$, respectively. Qualitatively, the results are similar to the extremal case and so these solutions are also stable under spherical perturbations.

Interestingly, the positivity of the effective potential seems to be generic. In figure 11, we present similar plots for a few solutions in a different theory with parameters $\nu=4$ and $\alpha=20$, where $Q^{*}=0.44721$. We consider $Q=1 / 2$ and so they are also thermodynamically stable. Each panel shows again three solutions with different values of the horizon temper- 
ature and, again, the effective potential is always positive definite. Scanning the space of solutions in various theories, it is always found that the positivity of the effective potential $U(x)$ is satisfied. This means that $\omega^{2}$ is positive and so the hairy black hole solutions, which are thermodynamically stable, are also stable against spherical perturbations.

\section{Discussion}

In this paper, we made a detailed examination of the thermodynamic stability of a general class of hairy charged black holes in flat spacetime. To obtain the results in a compact form for any value of the parameter $\nu$ in the dilaton potential, we have used a general criterion to check the relative signs of the relevant response functions (in both the grand-canonical and canonical ensemble) within well delimited regions of the corresponding phase space. Interestingly, we have found that there always exists a sub-class of stable black holes for which the heat capacity and permittivity are positive definite close to the extremality. Similar results were obtained previously in [1] for a particular case, $\nu \rightarrow \infty .{ }^{10}$ However, the phase structure is much richer when $\nu$ takes finite values.

In this case, there exists a new sub-class of thermodynamically stable configurations characterized by only one horizon (see section 4.5). Another new feature is the appearance of critical points in both ensembles. ${ }^{11}$ In grand-canonical ensemble, the stable configurations exist only when the chemical potential exceeds a critical value $\Phi_{0}$. In the canonical ensemble, though, the region of stability is constrained to temperatures lower than a critical temperature $T_{0}$. Graphically, the stable configurations are those located between the local minimum and the maximum of the isotherm curves in figure 19a. If $Q$ exceeds $\tilde{Q}$, then $\epsilon_{T}$ becomes negative even if $C_{Q}>0$ and so the thermodynamic stability is lost. One concrete example of this is shown in figure 12, where the black holes become unstable when the electric charge, $Q_{2}$, exceeds $\tilde{Q}$.

In addition to the thermodynamical stability, we have checked stability against spherically symmetric perturbations. For this, we have followed the standard procedure used before for other families of hairy charged black holes [9, 52-54]. After perturbing the metric, the electromagnetic field and the scalar field, we parameterize the spherical perturbations in terms of a master equation, a generalized tortoise coordinate, and an effective potential (5.4). The analysis of this effective potential reveals that the thermodynamically stable solutions are also stable under radial perturbations: all solutions analyzed (extremal and non-extremal) possess a regular and positive definite potential. Hence the spherical perturbations of these black holes are always damped exponentially with time. Let us also comment that, since the black holes that we have considered in this work are spherically symmetric, it is typically expected that perturbations on higher multipolar numbers will also be free of instabilities (see for instance examples of this in $[9,52,54,63,64]$ ), and it is reasonable to expect these configurations to be fully dynamically stable.

\footnotetext{
${ }^{10}$ This case is very special because the limit can not be taken directly in the general ansatz for the solution.

${ }^{11}$ The scalar fields in AdS also change the black hole behaviour in grand-canonical ensemble by generating swallowtail sections [73], a feature that is not present for Reissner-Nordström-AdS black hole [74].
} 

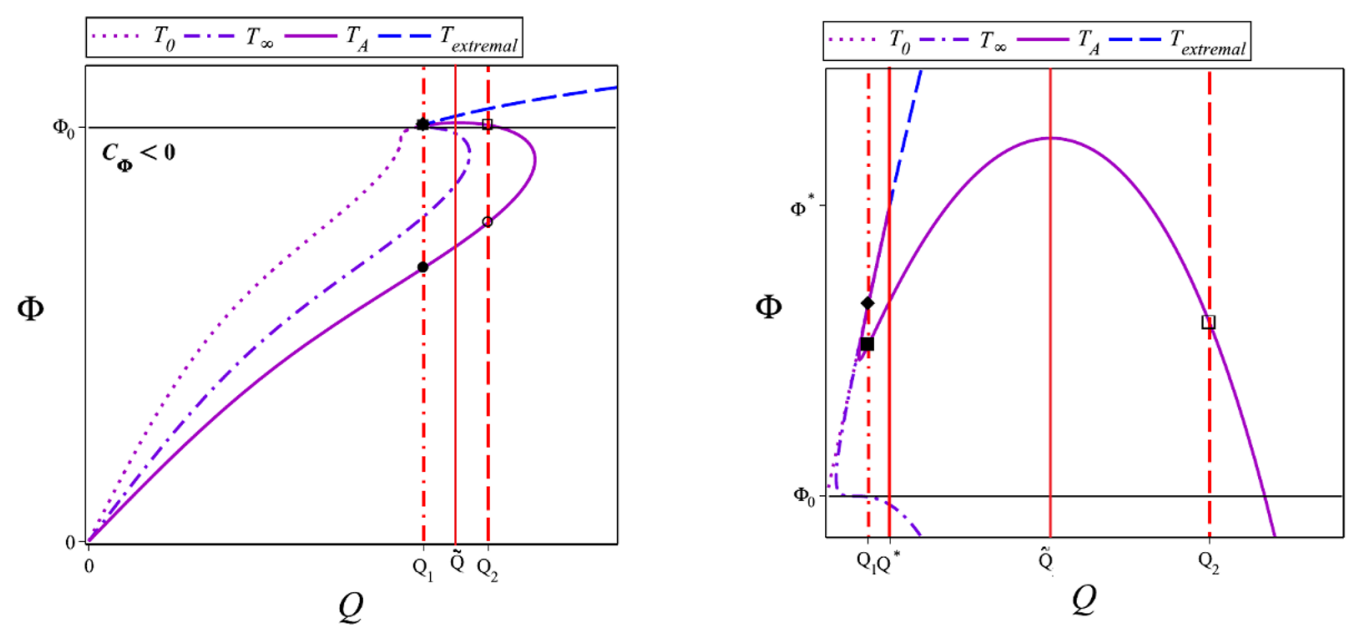

Figure 12. Right hand side: $Q_{2}>\tilde{Q}$, where the configuration at a $\Phi>\Phi_{0}$ becomes unstable $\left(\epsilon_{T}<0\right)$. Left hand side: the same image is zoomed in.

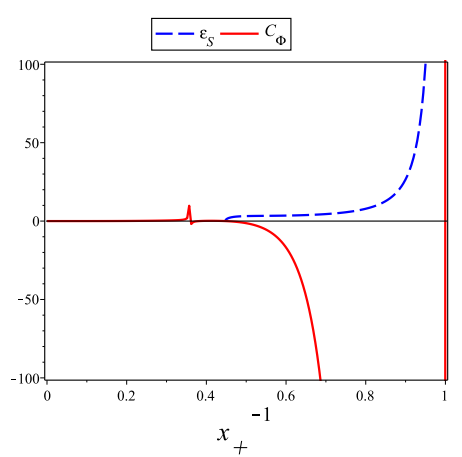

(a) $\Phi<\Phi_{0}$

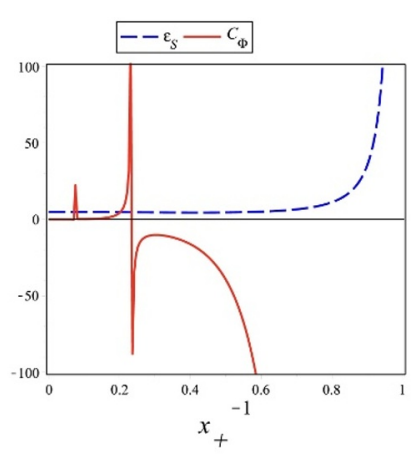

(b) $\Phi_{0}<\Phi<\Phi^{*}$

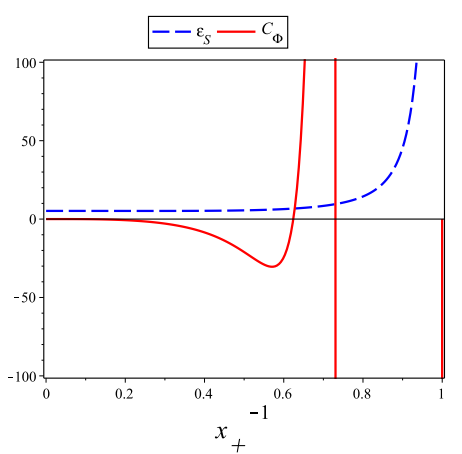

(c) $\Phi^{*}<\Phi$

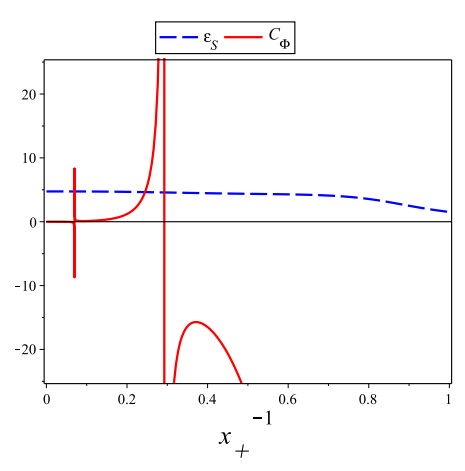

(d) $T<T_{\infty}$

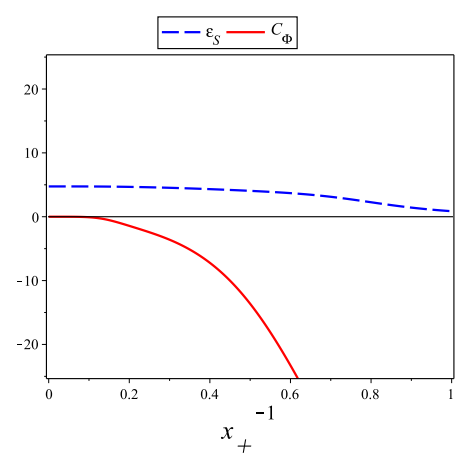

(e) $T_{\infty}<T$

Figure 13. Response functions $\epsilon_{S}$ y $C_{\Phi}$ vs the inverse of the horizon coordinate $x_{+}$(which accordingly is ranging the positive branch) for different values of the conjugate potential, as in (a), (b) and (c), and for temperature below and above the critical temperature $T_{\infty}$, as in (d) and (e).

For completeness, let us finally consider the particular case of $\nu=3$ and present, in figure 13 and figure 14, the plots of the response functions in a given ensemble with 


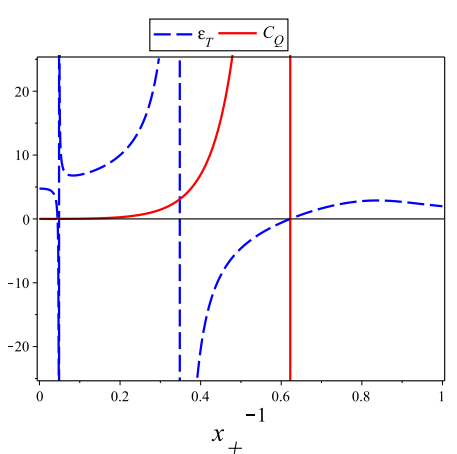

(a) $T<T_{\infty}$

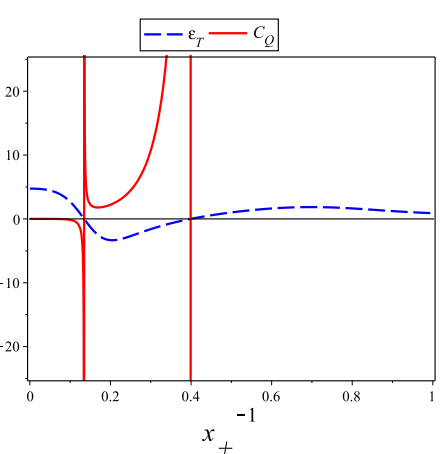

(b) $T_{\infty}<T<T_{0}$

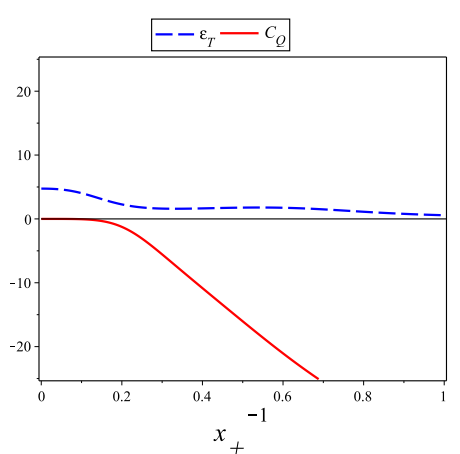

(c) $T_{0}<T$

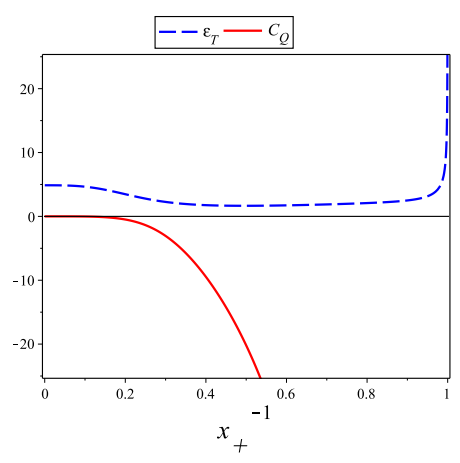

(d) $Q<Q_{0}$

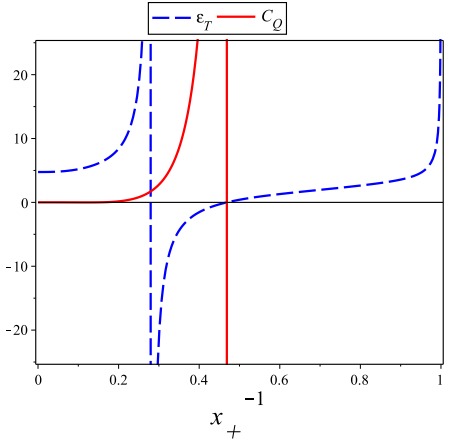

(e) $Q_{0}<Q$

Figure 14. Response functions $\epsilon_{T}$ y $C_{Q}$ vs the inverse of the horizon coordinate $x_{+}$for different values of the temperature, as in (a), (b) and (c), and for electric charge below and above the critical charge $Q_{0}$, as in (d) and (e).

respect to the location of the event horizon. We would like to directly check the existence of stability regions and to compare with the results obtained by the general criterion presented in section 3 and developed in appendix A.

Let us begin with the grand-canonical ensemble, where $\Phi$ is fixed. In this case, we observe that the thermodynamic stable black holes are found within two different intervals for the conjugate potential: $\Phi_{0}<\Phi<\Phi^{*}$ (see figure 13b) and $\Phi^{*}<\Phi$ (see figure 13c). The former corresponds to intermediate-size black holes between the two values of $x_{+}$where $C_{\Phi}$ is discontinuous. This region can also be identified in figure 15c where the slopes in $S$ vs $T$ are positive definite. This interesting new phase, which is not present in the case $\nu \rightarrow \infty$ studied previously in [1], contains configurations similar to Schwarzschild black hole in the sense that they have only a horizon. The region of stable black holes with $\Phi^{*}<\Phi$ can also be identified in figure 15c and, clearly, in this case there is a well defined extremal limit. As argued at the end of section 4 , stable black holes are found for $T<T_{\infty}$, as shown in figure 13d for a particular temperature in the interval $0<T<T_{\infty}$. Therefore, if $T_{\infty}<T$, there do not exist stable configurations, as shown in figure $13 \mathrm{e}$.

A similar analysis can be done for the canonical ensemble when $Q$ is fixed. First, we observe again that the stable black holes appear for $T<T_{\infty}$, as shown in figure 14a. This 
is consistent with figure $19 \mathrm{~b}$ for the isotherm $T_{A}<T_{\infty}$. However, for this isotherm, there exist two branches with $C_{Q}>0$ : one for $Q_{0}<Q<Q^{*}$ with solutions with one horizon and the other one for $Q^{*}<Q$ with stable black holes that have a well defined extremal limit $T=0$. This can also be observed in figure $14 \mathrm{e}$, where $Q_{0}<Q$. We remark that if $Q<Q_{0}$, there are no stable black holes.

To conclude, let us emphasize that not just the AdS arena stabilizes the thermodynamics of black holes, but also the existence of a dilaton potential in flat spacetime can have the same effect. As a first step, we have shown that the charged hairy black holes in flat space, besides being thermodynamically and perturbatively dynamically stable, have a rich phase structure including critical points as in AdS spacetime [74, 75]. We emphasize that the embedding in supergravity can be done only when the truncation contains two gauge fields [76] and so our results are valid in the limit when the strength of one gauge field is much weaker than the other one.

\section{Acknowledgments}

We would like to thank Andrés Anabalón, David Choque, Carlos Herdeiro, Jutta Kunz, Robert Mann, and Eugen Radu for interesting discussions at various stages of this work and collaboration on related projects. We also thank the anonymous referee for interesting comments and suggestions. The research of DA is supported by the Fondecyt Grants 1200986, 1170279, 1171466, and 2019/13231-7 Programa de Cooperacion Internacional, ANID. JLBS gratefully acknowledges support from DFG Project No. BL 1553, DFG Research Training Group 1620 Models of Gravity, the COST Action CA16104 GWverse, and the FCT projects PTDC/FISOUT/28407/2017 and PTDC/FIS-AST/3041/2020. The research of RR was partially supported by the Ph.D. scholarship CONICYT (now succeeded by ANID) 21140024.

\section{A Thermodynamic stability analysis for arbitrary $\nu$}

In this section, we present a detailed analysis of local thermodynamic stability for an arbitrary value of $\nu$ by following the general criterion proposed in section 3.2. The main result is that there exists a sub-class of asymptotically flat hairy black holes that are thermodynamically stable in both ensembles and for every finite value of the hairy parameter $\nu$.

In what follows we will be referring to the behaviour of the thermodynamical quantities already shown in section 3.3 .

\section{A.1 Grand-canonical ensemble, $\Phi$ fixed}

The relevant quantities required to study the thermodynamic stability are the electric permittivity at constant entropy, $\epsilon_{S}=(\partial \Phi / \partial Q)_{S}$, and the heat capacity at constant conjugate potential, $C_{\Phi}=T(\partial S / \partial T)_{\Phi}$. We are going to read off the signs of these response functions by studying general properties of the corresponding phase diagrams. 


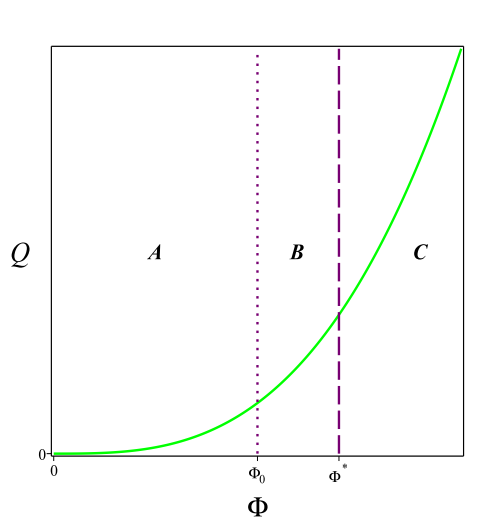

(a)

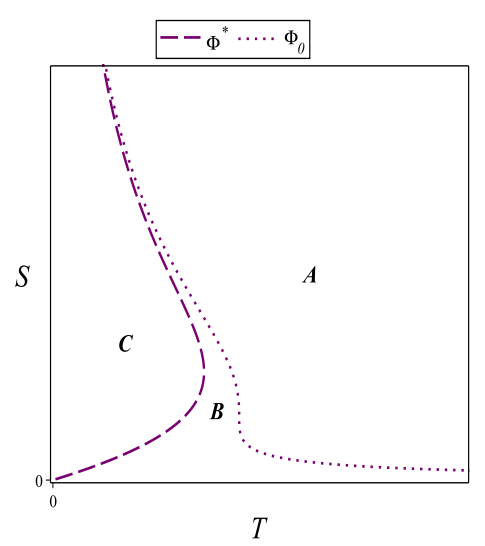

(b)

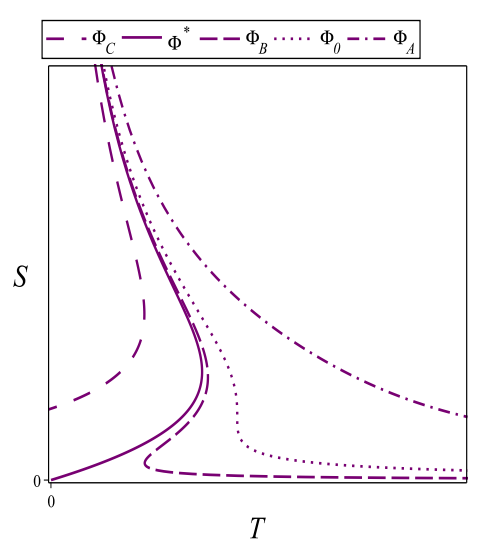

(c) $\Phi_{A}<\Phi_{0}<\Phi_{B}<\Phi^{*}<\Phi_{C}$

Figure 15. (a) Sketch of $Q$ vs $\Phi$ at fixed entropy. (b) Relevant curves in the $S$ vs $T$ diagram. (c) Sketch of $S$ vs $T$ for some relevant values of the conjugate potential.

\section{A.1.1 $Q$ vs $\Phi, S$ fixed diagram}

The general parametric equations of the thermodynamical quantities of interest are found in equation (3.8). As it has already been mentioned it above, the asymptotic limits are straightforward to compute and the result is that in the behaviour of the curves in the $Q$ vs $\Phi$ diagram with fixed entropy must be such that they must start at the origin and then diverge as one of the to quantities diverges. This does not tell us anything about the behaviour for finite values of $Q$ or $\Phi$. However, one can show that there are no points for which one or both of the following equations are satisfied

$$
\left(\frac{\partial \Phi}{\partial Q}\right)_{S}=0, \quad\left(\frac{\partial^{2} \Phi}{\partial Q^{2}}\right)_{S}=0
$$

which allows us to conclude that $\epsilon_{S}$ is a positive definite quantity for all equilibrium configurations. A sketch for $Q$ vs $\Phi$ at constant $S$, which holds for any value of $\nu>1$, is presented in figure 15a.

\section{A.1.2 $S$ vs $T, \Phi$ fixed diagram}

The relevant expressions in this case are the ones in equation (3.10). To facilitate the analysis we recall that the study of the limits $x_{+}=1$ and $x_{+} \rightarrow \infty$ on the expressions of entropy and temperature above yields the following results:

$$
\begin{aligned}
& \lim _{x_{+} \rightarrow+\infty} S=0, \quad \lim _{x_{+} \rightarrow+\infty} T \rightarrow\{-\infty, 0,+\infty\}, \\
& \lim _{x_{+} \rightarrow 1^{+}} S \rightarrow+\infty, \quad \lim _{x_{+} \rightarrow 1^{+}} T=0
\end{aligned}
$$

We emphasize that the physical analysis of these results is made only for positive values of the temperature. However, the existence of a divergent negative temperature indicates that there also exists a configuration with zero temperature, which corresponds to an 
extremal black hole. In particular, the $\lim _{x_{+} \rightarrow+\infty} T=-\infty$ is consistent only when $\Phi>\Phi^{*}$. Therefore, it means that there must exist extremal black holes with non-zero entropy in this interval, as can be explicitly checked in figure 15c. Indeed, the curve characterized by $\Phi_{C}>\Phi^{*}$ reaches the extremality at finite entropy.

The above result, together with the existence of a critical point (see figure 1a) allow us to sketch the general behaviour of the system in this case as shown in figure $15 \mathrm{~b}$ and $15 \mathrm{c}$.

\section{A.1.3 Thermodynamic stability}

Armed with the information obtained in the previous analysis, it is useful to consider three regions (noted A, B, and $\mathrm{C}$, respectively) where the heat capacity $C_{\Phi}$ changes its sign in a particular way. Those regions are identified by some specific values of $\Phi$, as shown in figure 15a. Since $\epsilon_{S}>0$, the thermodynamic stability for black holes in grand-canonical ensemble is basically guaranteed provided $C_{\Phi}>0$.

Region $A$ with $\Phi<\Phi_{0}$ : in this region, $C_{\Phi}<0$, as can be seen from figure 15b, implying that there are no thermodynamically stable equilibrium configurations.

Regions $B$ with $\Phi_{0}<\Phi<\Phi^{*}$ and region $C$ with $\Phi^{*}<\Phi$ : since $C_{\Phi}$ changes its sign at least one time, there must exist at least one region where $C_{\Phi}>0$. Accordingly, we conclude that thermodynamically stable hairy black holes can be found in the range $2>\Phi \geq \Phi_{0}$.

\section{A.2 Canonical ensemble, $Q$ fixed}

The relevant quantities are the electric permittivity at constant temperature, $\epsilon_{T} \equiv$ $(\partial \Phi / \partial Q)_{T}$, and the heat capacity, $C_{Q} \equiv T(\partial S / \partial T)_{Q}$. As before, we are going to use the corresponding diagrams to read off the sign of the slopes and, accordingly, split the parameter space into different regions.

\section{A.2.1 $\Phi$ vs $Q, T$ fixed}

By using the equations (3.16), we recall their relevant limits which are

$$
\begin{array}{rlrl}
\Phi^{*}:= & \left.\lim _{x_{+} \rightarrow+\infty} \Phi\right|_{T=0} & =\sqrt{2}, & Q^{*}:= \\
\left.\lim _{x_{+} \rightarrow 1^{+}} \Phi\right|_{T=0} & =2, & \left.\lim _{x++\infty} Q\right|_{T=0} & =\frac{\nu \sqrt{2} \sqrt{\nu+2}}{4 \sqrt{\nu-1}}, \\
\left.\lim _{x_{+} \rightarrow 1^{+}} Q\right|_{T=0} \rightarrow+\infty .
\end{array}
$$

For non-extremal black holes $T \neq 0$, the limit $x_{+} \rightarrow+\infty$ gives the same result, which means that the point $\left(Q^{*}, \Phi^{*}\right)$ is an end point for all the isotherms, while the limit $x_{+} \rightarrow 1$ yields $(Q, \Phi) \rightarrow(0,0)$, as shown also in figure 16a. The critical temperatures $T_{0}$ and $T_{\infty}$, defined in table 1 , allow us to split the phase space into relevant regions which will be used to compare the relative signs of the electric permittivity $\epsilon_{T}$ and heat capacity $C_{Q}$. It is also worth re-emphasizing that $T_{\infty}$ and $T_{0}$ depend on $\nu$, as can be seen in figure 1a. 


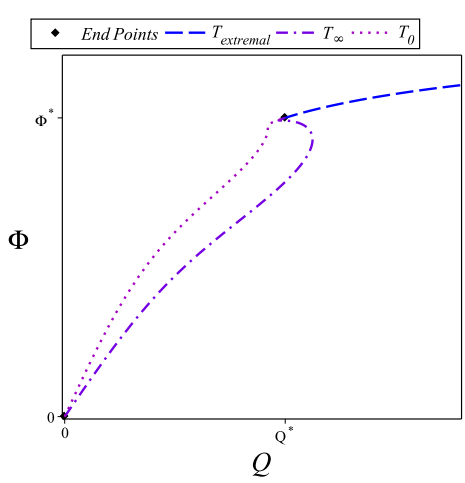

(a) $0=T_{\text {extremal }}<T_{0}<T_{\infty}$

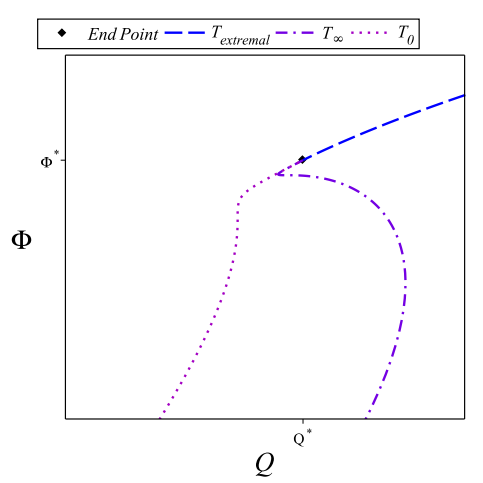

(b) Zoom-in of (a)

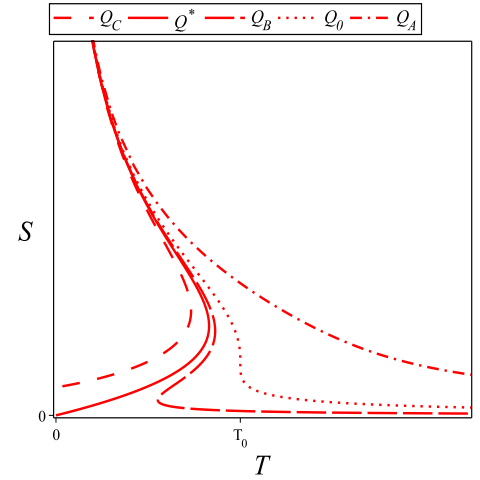

(c) $Q_{A}<Q_{0}<Q_{B}<Q^{*}<Q_{C}$

Figure 16. (a) Sketch of the relevant isotherms $\Phi$ vs $Q$. (b) Zoom near one end point for the isotherms. (c) Sketch of $S$ vs $T$ for some relevant values of the charge.

\section{A.2.2 $S$ vs $T, Q$ fixed}

In comparison with the case with fixed $\Phi$, the equations (3.22) for fixed charge produce exactly the same limits as before, just with a different critical temperature $T_{0}$. So the behaviour should be exactly the same. A sketch is depicted in figure 16c with the relevant quantities that belong to this case. With respect to the stability, while it is clear that $C_{Q}<0$ for all $Q<Q_{0}$, the case $Q>Q_{0}$ must be carefully analyzed.

\section{A.2.3 Thermodynamic stability}

To begin the analysis of thermodynamic stability, first note that $T_{0}$ and $Q_{0}$ define a critical point (their definitions are provided in table 1 ). This can be checked by inserting $T_{0}$ into the equation for $Q$ in equation (3.16). This observation makes possible to identify in a practical and direct manner the regions where the response functions are positively defined, as it is shown in figure 1a and figure $1 \mathbf{b}$. Before presenting the details of the analysis, we would like to emphasize that by comparing the number of times each response function changes its sign in a specific region, we can extract important information with the help of figure 1; indeed, we can conclude immediately that the relevant intervals for the thermodynamic stability are $T<T_{\infty}$ and $Q>Q_{0}$. However, we have to make sure that all regions with unstable configurations are excluded and now we investigate this issue case by case (see figure 17).

Regions IA, IB, IC: these regions are characterized by $Q<Q_{0}$. The electric permittivity is positive, but the heat capacity is negative and so all the configurations are thermodynamically unstable.

Regions IIA, IIC: within these two regions, characterized by $Q_{0}<Q<Q^{*}$ and $T>T_{0}$ (for IIA) and $T<T_{\infty}$ (for IIC), we observe that $\epsilon_{T}>0$ but $C_{Q}<0$, therefore, there are no thermodynamically stable black holes. 


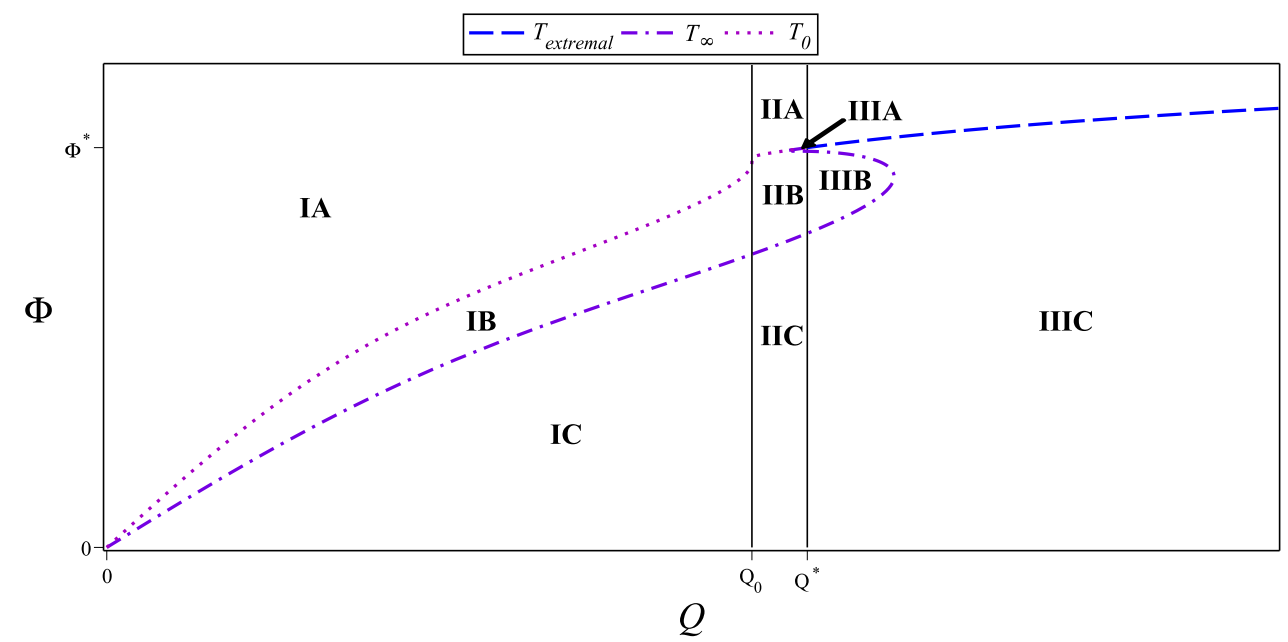

(a) The phase space $S$ vs $T$ separated into the relevant regions

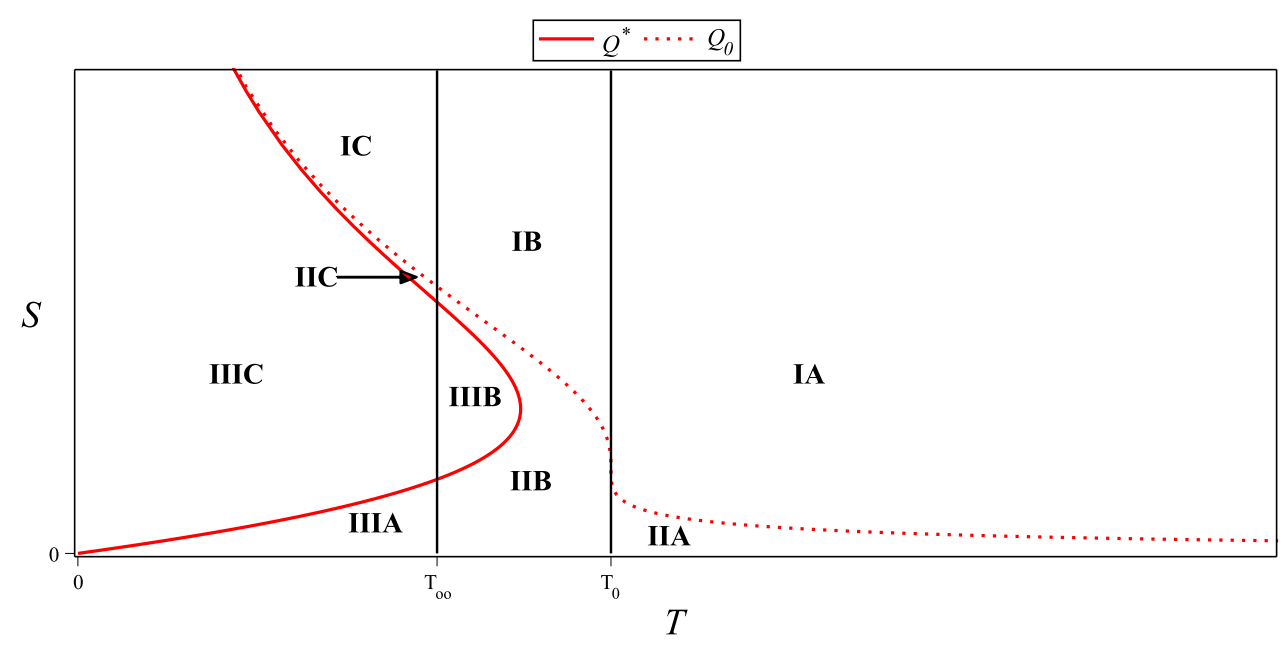

(b) The phase space $S$ vs $T$ separated into the relevant regions

Figure 17. The phase spaces: (a) $\Phi$ vs $Q$ and (b) $S$ vs $T$.

Region IIB: in this region, characterized by $Q_{0}<Q<Q^{*}$ and $T_{\infty}<T<T_{0}$, the response functions change two times their signs. Despite that, it is not straightforward to determine whether both are positive for a given configuration, as required for thermodynamic stability. We should discuss further this case below.

Region IIIA: inside this region, $C_{Q}$ changes one time its sign, while $\epsilon_{T}$ changes its sign at least one time. Again, this is not sufficient to prove that both of them are positive for a given configuration and we investigate this case below.

Region IIIB: since both response functions change their signs only one time, one can not conclude about the stability. We shall comment on this case below. 


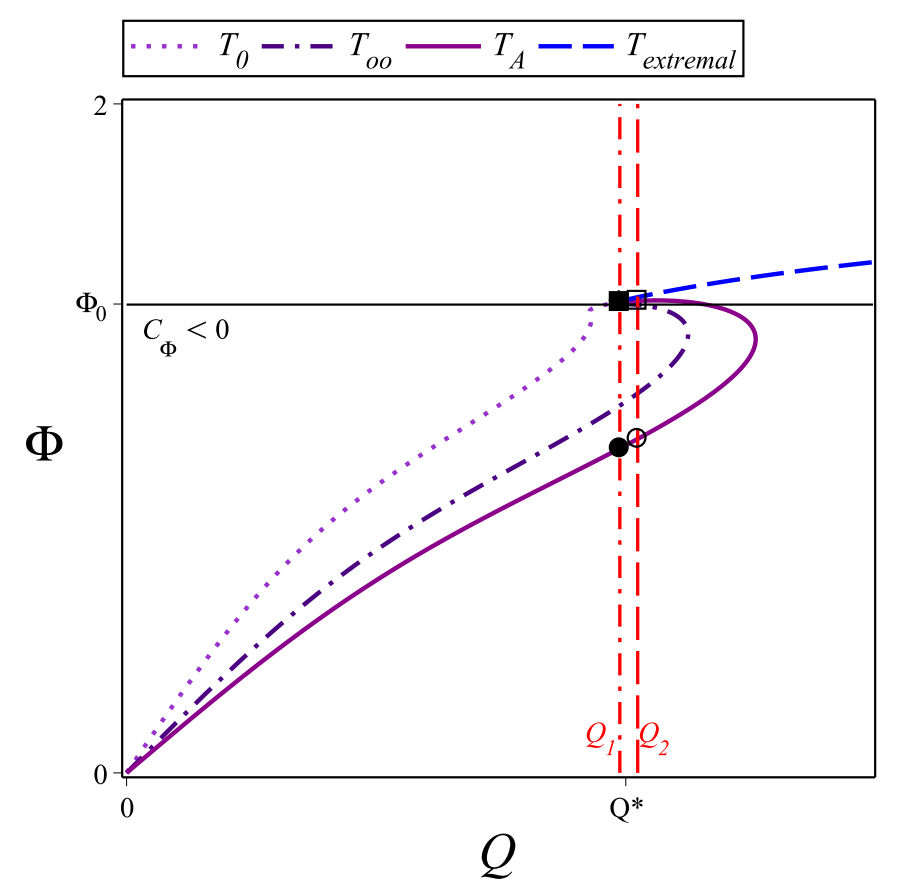

Figure 18. $\Phi$ vs $Q$ at fixed $T$. The relevant isotherms are $T_{0}$ and $T_{\infty}$. An arbitrary isotherm $0<T_{A}<T_{\infty}$ is traced. Two arbitrary isocharges, $Q_{1}<Q^{*}$ and $Q^{*}<Q_{2}$, and their intersections with $T_{A}$ was marked. The line $Q=Q_{1}$ intersects three times $T_{A}$ (the two intersections at the top cannot be distinguished at this scale. See figure 19a for a zoomed image). The line $Q=Q_{2}$ intersects two times $T_{A}$.

Region IIIC: inside this region, $\epsilon_{T}$ can change its sign at least one time, while the sign of $C_{Q}$ changes only one time. That is not sufficient to prove that both of them are positive for a given configuration. We shall comment on this case right below.

So far, we can definitely conclude that the regions IA, IB, IC, IIA and IIC do not contain locally stable configurations. Now, to complete the analysis, it will be useful to consider the following thermodynamic relation:

$$
C_{\Phi}=C_{Q}+\epsilon_{T} \alpha_{Q}^{2} T
$$

r where $\alpha_{Q} \equiv(\partial \Phi / \partial T)_{Q}$. From the results presented in section A.1 for the grand-canonical ensemble, we know that $C_{\Phi}<0$ as long as $\Phi<\Phi_{0}$, which implies that either $C_{Q}<0$ or $\epsilon_{T}<0$. For $\Phi_{0}<\Phi$, there is a sub-region within IIB where one of the three configurations at a given $T_{\infty}<T<T_{0}$ has $C_{\Phi}>0$ (see figure 17b), but it has also $\epsilon_{T}<0$ as inferred after drawing the corresponding isotherm in figure 17a. Therefore, the regions IIB and IIIB contain no thermodynamically stable configurations. This is better appreciated in figure 18, where the line $\Phi=\Phi_{0}$ was explicitly marked.

In what follows, we are going to consider only the cases $\Phi>\Phi_{0}$, corresponding to the remaining regions to be analized: IIIA and IIIC. The equation (A.2) does not provide enough information to conclude about the stability in the case $\Phi>\Phi_{0}$. The reason is that $C_{\Phi}$ is not always positive inside this interval. In fact, inside both regions IIIA and IIIC, 


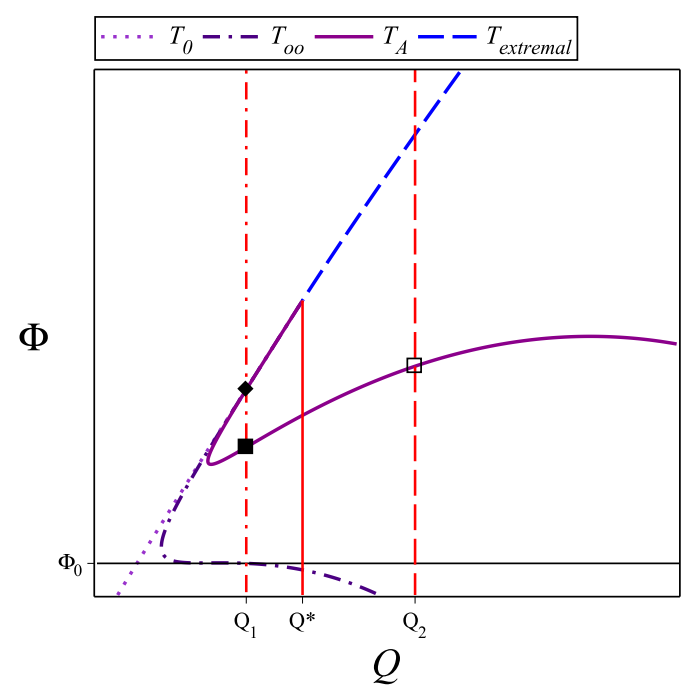

(a) Zoom to the stability region (see figure 18)

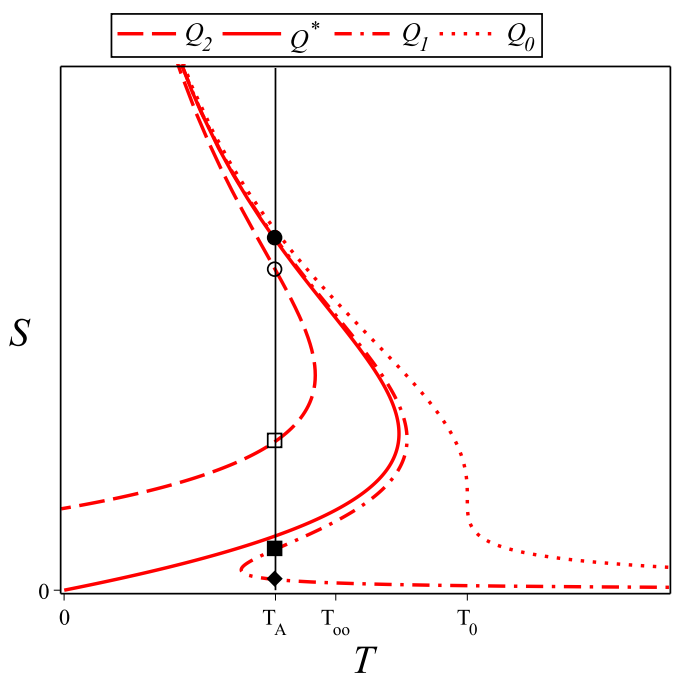

(b) $Q_{0}<Q_{1}<Q^{*}<Q_{2}$

Figure 19. (a) A closer look into the region of stability. The intersections between $Q_{1}, Q_{2}$ and $T_{A}$ are represented with different symbols and, in (b), it is shown the some intersection points by the same symbols. (b) $S$ vs $T$ at fixed $Q$; it is shown the intersection between $T_{A}$ with $Q_{1}$ and $Q_{2}$.

the heat capacity can have negative or positive values (see figures 18 and 19b, where two curves at constant charges, $Q_{1}$ and $Q_{2}$, are plotted). Then, in order to determine whether these regions contain thermodynamic stable configurations, let us consider the isotherm $T_{A}<T_{\infty}$ depicted in figure 18 and magnified in figure 19a. If $Q<Q^{*}$, the isotherm $T_{A}$ represents the region IIIA and, if $Q>Q^{*}$, represents region IIIC, as follows from figure 17a. ${ }^{12}$ That is why we have considered two isocharge lines, $Q_{1}$ and $Q_{2}$, so that $Q_{0}<Q_{1}<Q^{*}$ and $Q^{*}<Q_{2}$. The isotherm $T_{A}$ intersects three times the isocharge line $Q_{1}$ and two times the isocharge line corresponding to $Q_{2}$.

Let us focus first on the three intersection points between the curves characterized by $Q_{1}$ and $T_{A}$. We have used three different symbol for each intersection: a filled diamond (with the highest value of $\Phi$ in $\Phi$ vs $Q$ and the lowest value of $S$ in $S$ vs $T$ ), a filled squared (with an intermediate value of $\Phi$ and an intermediate value of $S$, respectively), and a filled circle (with lowest $\Phi$ and highest $S$, respectively). From figure 19, it follows that, indeed, the branch represented by the filled squared (located within IIIA) contains configurations with both $\epsilon_{T}>0$ and $C_{Q}>0$. These thermodynamically stable black holes, however, do not have an extremal limit, because the region $Q<Q^{*}$ does not contain extremal black holes.

Let us now consider $T_{A}$ and an isocharge line $Q_{2}>Q^{*}$. From the plot $S$ vs $T$ it is clear that there are two branches of configurations and that one of them has $C_{Q}>0$. Interestingly, in the plot $\Phi$ vs $Q, Q_{2}$ intersects $T_{A}$ for two configurations. If $Q_{2}$ is close enough to $Q^{*}$, then the two intersections correspond to branches where $\epsilon_{T}>0$. Therefore, inside this region, contained within IIIC, there are also thermodynamically stable black holes which, in addition, have a well defined extremal limit.

\footnotetext{
${ }^{12}$ The region IIIA is a tiny region which is better appreciated in figure 19a.
} 
Since the charge $Q_{1}$ is arbitrary and the temperature $T_{A}$ is just limited by the condition $T_{A}<T_{\infty}$, we can certainly conclude that the most general region of stability in the plot of $\Phi-Q$ at constant $T$ is found between the local minimum and maximum of every isotherm such that $T<T_{\infty}$ (see figure 19a), namely, inside a subset of both the region IIIA and IIIC.

Open Access. This article is distributed under the terms of the Creative Commons Attribution License (CC-BY 4.0), which permits any use, distribution and reproduction in any medium, provided the original author(s) and source are credited.

\section{References}

[1] D. Astefanesei, D. Choque, F. Gómez and R. Rojas, Thermodynamically stable asymptotically flat hairy black holes with a dilaton potential, JHEP 03 (2019) 205 [arXiv: 1901.01269] [INSPIRE].

[2] J.D. Bekenstein, Black holes and entropy, Phys. Rev. D 7 (1973) 2333 [InSPIRE].

[3] S.W. Hawking, Particle Creation by Black Holes, Commun. Math. Phys. 43 (1975) 199 [Erratum ibid. 46 (1976) 206] [INSPIRE].

[4] S.W. Hawking, Black Holes and Thermodynamics, Phys. Rev. D 13 (1976) 191 [InSPIRE].

[5] J.W. York, Jr., Black hole thermodynamics and the Euclidean Einstein action, Phys. Rev. D 33 (1986) 2092 [INSPIRE].

[6] A. Ishibashi and R.M. Wald, Dynamics in nonglobally hyperbolic static space-times. 3. Anti-de Sitter space-time, Class. Quant. Grav. 21 (2004) 2981 [hep-th/0402184] [INSPIRE].

[7] S.W. Hawking and D.N. Page, Thermodynamics of Black Holes in anti-de Sitter Space, Commun. Math. Phys. 87 (1983) 577 [InSPIRE].

[8] A. Anabalon, D. Astefanesei and R. Mann, Exact asymptotically flat charged hairy black holes with a dilaton potential, JHEP 10 (2013) 184 [arXiv:1308.1693] [INSPIRE].

[9] D. Astefanesei, J.L. Blázquez-Salcedo, C. Herdeiro, E. Radu and N. Sanchis-Gual, Dynamically and thermodynamically stable black holes in Einstein-Maxwell-dilaton gravity, JHEP 07 (2020) 063 [arXiv: 1912.02192] [INSPIRE].

[10] C.A.R. Herdeiro and E. Radu, Asymptotically flat black holes with scalar hair: a review, Int. J. Mod. Phys. D 24 (2015) 1542014 [arXiv: 1504.08209] [INSPIRE].

[11] D. Sudarsky, A Simple proof of a no hair theorem in Einstein Higgs theory,, Class. Quant. Grav. 12 (1995) 579 [inSPIRE].

[12] J.D. Bekenstein, Novel "no-scalar-hair" theorem for black holes, Phys. Rev. D 51 (1995) 6608 [INSPIRE].

[13] M. Henneaux, C. Martinez, R. Troncoso and J. Zanelli, Black holes and asymptotics of $2+1$ gravity coupled to a scalar field, Phys. Rev. D 65 (2002) 104007 [hep-th/0201170] [INSPIRE].

[14] C. Martinez, R. Troncoso and J. Zanelli, Exact black hole solution with a minimally coupled scalar field, Phys. Rev. D 70 (2004) 084035 [hep-th/0406111] [INSPIRE].

[15] A. Anabalon, Exact Black Holes and Universality in the Backreaction of non-linear $\sigma$-models with a potential in (A)dS4, JHEP 06 (2012) 127 [arXiv:1204.2720] [INSPIRE]. 
[16] A. Acena, A. Anabalon and D. Astefanesei, Exact hairy black brane solutions in AdS $S_{5}$ and holographic RG flows, Phys. Rev. D 87 (2013) 124033 [arXiv:1211.6126] [INSPIRE].

[17] A. Anabalón and D. Astefanesei, On attractor mechanism of AdS 4 black holes, Phys. Lett. B 727 (2013) 568 [arXiv:1309.5863] [INSPIRE].

[18] A. Anabalon, D. Astefanesei and C. Martinez, Mass of asymptotically anti-de Sitter hairy spacetimes, Phys. Rev. D 91 (2015) 041501 [arXiv:1407.3296] [InSPIRE].

[19] A. Anabalon, D. Astefanesei, D. Choque and C. Martinez, Trace Anomaly and Counterterms in Designer Gravity, JHEP 03 (2016) 117 [arXiv: 1511.08759] [INSPIRE].

[20] X.-H. Feng, H. Lü and Q. Wen, Scalar Hairy Black Holes in General Dimensions, Phys. Rev. D 89 (2014) 044014 [arXiv: 1312.5374] [INSPIRE].

[21] H.-S. Liu and H. Lü, Scalar Charges in Asymptotic AdS Geometries, Phys. Lett. B 730 (2014) 267 [arXiv:1401.0010] [InSPIRE].

[22] H. Lü, C.N. Pope and Q. Wen, Thermodynamics of AdS Black Holes in Einstein-Scalar Gravity, JHEP 03 (2015) 165 [arXiv:1408.1514] [INSPIRE].

[23] G.W. Gibbons, Antigravitating Black Hole Solitons with Scalar Hair in N=4 Supergravity, Nucl. Phys. B 207 (1982) 337.

[24] G.W. Gibbons and K.-i. Maeda, Black Holes and Membranes in Higher Dimensional Theories with Dilaton Fields, Nucl. Phys. B 298 (1988) 741 [InSPIRE].

[25] D. Garfinkle, G.T. Horowitz and A. Strominger, Charged black holes in string theory, Phys. Rev. D 43 (1991) 3140 [Erratum ibid. 45 (1992) 3888] [INSPIRE].

[26] K. Goldstein, N. Iizuka, R.P. Jena and S.P. Trivedi, Non-supersymmetric attractors, Phys. Rev. D 72 (2005) 124021 [hep-th/0507096] [INSPIRE].

[27] D. Astefanesei, C. Herdeiro, A. Pombo and E. Radu, Einstein-Maxwell-scalar black holes: classes of solutions, dyons and extremality, JHEP 10 (2019) 078 [arXiv: 1905.08304] [INSPIRE].

[28] A. Anabalón, D. Astefanesei, A. Gallerati and M. Trigiante, Hairy Black Holes and Duality in an Extended Supergravity Model, JHEP 04 (2018) 058 [arXiv:1712.06971] [INSPIRE].

[29] U. Nucamendi and M. Salgado, Scalar hairy black holes and solitons in asymptotically flat space-times, Phys. Rev. D 68 (2003) 044026 [gr-qc/0301062] [INSPIRE].

[30] J.D. Brown and J.W. York, Jr., Quasilocal energy and conserved charges derived from the gravitational action, Phys. Rev. D 47 (1993) 1407 [gr-qc/9209012] [INSPIRE].

[31] S.R. Lau, Light cone reference for total gravitational energy, Phys. Rev. D 60 (1999) 104034 [gr-qc/9903038] [INSPIRE].

[32] R.B. Mann, Misner string entropy, Phys. Rev. D 60 (1999) 104047 [hep-th/9903229] [INSPIRE].

[33] P. Kraus, F. Larsen and R. Siebelink, The gravitational action in asymptotically AdS and flat space-times, Nucl. Phys. B 563 (1999) 259 [hep-th/9906127] [INSPIRE].

[34] R.B. Mann and D. Marolf, Holographic renormalization of asymptotically flat spacetimes, Class. Quant. Grav. 23 (2006) 2927 [hep-th/0511096] [INSPIRE].

[35] D. Astefanesei and E. Radu, Quasilocal formalism and black ring thermodynamics, Phys. Rev. D 73 (2006) 044014 [hep-th/0509144] [INSPIRE]. 
[36] D. Astefanesei, R.B. Mann and C. Stelea, Note on counterterms in asymptotically flat spacetimes, Phys. Rev. D 75 (2007) 024007 [hep-th/0608037] [INSPIRE].

[37] D. Astefanesei, R.B. Mann, M.J. Rodriguez and C. Stelea, Quasilocal formalism and thermodynamics of asymptotically flat black objects, Class. Quant. Grav. 27 (2010) 165004 [arXiv:0909.3852] [INSPIRE].

[38] G. Compere, F. Dehouck and A. Virmani, On Asymptotic Flatness and Lorentz Charges, Class. Quant. Grav. 28 (2011) 145007 [arXiv:1103.4078] [INSPIRE].

[39] G. Compere and F. Dehouck, Relaxing the Parity Conditions of Asymptotically Flat Gravity, Class. Quant. Grav. 28 (2011) 245016 [Erratum ibid. 30 (2013) 039501] [arXiv:1106. 4045] [INSPIRE].

[40] D. Astefanesei, M.J. Rodriguez and S. Theisen, Thermodynamic instability of doubly spinning black objects, JHEP 08 (2010) 046 [arXiv: 1003.2421] [INSPIRE].

[41] R.L. Arnowitt, S. Deser and C.W. Misner, Canonical variables for general relativity, Phys. Rev. 117 (1960) 1595 [INSPIRE].

[42] R. Arnowitt, S. Deser and C.W. Misner, Energy and the Criteria for Radiation in General Relativity, Phys. Rev. 118 (1960) 1100 [INSPIRE].

[43] R.L. Arnowitt, S. Deser and C.W. Misner, Coordinate invariance and energy expressions in general relativity, Phys. Rev. 122 (1961) 997 [INSPIRE].

[44] R.L. Arnowitt, S. Deser and C.W. Misner, The Dynamics of general relativity, Gen. Rel. Grav. 40 (2008) 1997 [gr-qc/0405109] [INSPIRE].

[45] D. Astefanesei, R. Ballesteros, D. Choque and R. Rojas, Scalar charges and the first law of black hole thermodynamics, Phys. Lett. B 782 (2018) 47 [arXiv:1803.11317] [INSPIRE].

[46] R. Rojas Mejías, Thermodynamics of Asymptotically Flat Dyonic Black Holes, Phys. Rev. D 101 (2020) 124030 [arXiv:1907.10681] [INSPIRE].

[47] H.B. Callen, Thermodynamics and an Introduction to Thermostatistics, John Wiley and Sons, New York U.S.A. (1985).

[48] G.W. Gibbons, R. Kallosh and B. Kol, Moduli, scalar charges, and the first law of black hole thermodynamics, Phys. Rev. Lett. 77 (1996) 4992 [hep-th/9607108] [INSPIRE].

[49] K. Hajian and M.M. Sheikh-Jabbari, Redundant and Physical Black Hole Parameters: Is there an independent physical dilaton charge?, Phys. Lett. B $\mathbf{7 6 8}$ (2017) 228 [arXiv: 1612.09279] [INSPIRE].

[50] F. Naderi and A. Rezaei-Aghdam, New five-dimensional Bianchi type magnetically charged hairy topological black hole solutions in string theory, Eur. Phys. J. C 79 (2019) 995 [arXiv: 1905.11302] [INSPIRE].

[51] D. Astefanesei, F. Gómez, J. Maggiolo and R. Rojas, Critical behaviour for asymptotically flat hairy black holes, to appear.

[52] J.L. Blázquez-Salcedo, S. Kahlen and J. Kunz, Quasinormal modes of dilatonic Reissner-Nordström black holes, Eur. Phys. J. C 79 (2019) 1021 [arXiv:1911.01943] [INSPIRE].

[53] J.L. Blázquez-Salcedo, C.A.R. Herdeiro, J. Kunz, A.M. Pombo and E. Radu, Einstein-Maxwell-scalar black holes: the hot, the cold and the bald, Phys. Lett. B 806 (2020) 135493 [arXiv: 2002.00963] [INSPIRE]. 
[54] J.L. Blázquez-Salcedo, C.A.R. Herdeiro, S. Kahlen, J. Kunz, A.M. Pombo and E. Radu, Quasinormal modes of hot, cold and bald Einstein-Maxwell-scalar black holes, Eur. Phys. J. C 81 (2021) 155 [arXiv: 2008.11744] [INSPIRE].

[55] E.W. Leaver, Quasinormal modes of Reissner-Nordstrom black holes, Phys. Rev. D 41 (1990) 2986 [INSPIRE].

[56] V. Ferrari, M. Pauri and F. Piazza, Quasinormal modes of charged, dilaton black holes, Phys. Rev. D 63 (2001) 064009 [gr-qc/0005125] [INSPIRE].

[57] Y.S. Myung and D.-C. Zou, Instability of Reissner-Nordström black hole in Einstein-Maxwell-scalar theory, Eur. Phys. J. C 79 (2019) 273 [arXiv:1808.02609] [INSPIRE].

[58] Y.S. Myung and D.-C. Zou, Quasinormal modes of scalarized black holes in the Einstein-Maxwell-Scalar theory, Phys. Lett. B 790 (2019) 400 [arXiv:1812.03604] [INSPIRE].

[59] R. Brito and C. Pacilio, Quasinormal modes of weakly charged Einstein-Maxwell-dilaton black holes, Phys. Rev. D 98 (2018) 104042 [arXiv: 1807.09081] [InSPIRE].

[60] A. Jansen, A. Rostworowski and M. Rutkowski, Master equations and stability of Einstein-Maxwell-scalar black holes, JHEP 12 (2019) 036 [arXiv:1909.04049] [INSPIRE].

[61] J.L. Blázquez-Salcedo, D.D. Doneva, J. Kunz and S.S. Yazadjiev, Radial perturbations of the scalarized Einstein-Gauss-Bonnet black holes, Phys. Rev. D 98 (2018) 084011 [arXiv: 1805.05755] [INSPIRE].

[62] A. Anabalon and N. Deruelle, On the mechanical stability of asymptotically flat black holes with minimally coupled scalar hair, Phys. Rev. D 88 (2013) 064011 [arXiv:1307.2194] [INSPIRE].

[63] J.L. Blázquez-Salcedo, D.D. Doneva, S. Kahlen, J. Kunz, P. Nedkova and S.S. Yazadjiev, Axial perturbations of the scalarized Einstein-Gauss-Bonnet black holes, Phys. Rev. D 101 (2020) 104006 [arXiv:2003.02862] [INSPIRE].

[64] J.L. Blázquez-Salcedo, D.D. Doneva, S. Kahlen, J. Kunz, P. Nedkova and S.S. Yazadjiev, Polar quasinormal modes of the scalarized Einstein-Gauss-Bonnet black holes, Phys. Rev. D 102 (2020) 024086 [arXiv:2006.06006] [INSPIRE].

[65] J.L. Blázquez-Salcedo, F.S. Khoo and J. Kunz, Quasinormal modes of Einstein-Gauss-Bonnet-dilaton black holes, Phys. Rev. D 96 (2017) 064008 [arXiv: 1706.03262] [INSPIRE].

[66] J.L. Blázquez-Salcedo et al., Perturbed black holes in Einstein-dilaton-Gauss-Bonnet gravity: Stability, ringdown, and gravitational-wave emission, Phys. Rev. D 94 (2016) 104024 [arXiv: 1609.01286] [INSPIRE].

[67] T. Torii and K.-i. Maeda, Stability of a dilatonic black hole with a Gauss-Bonnet term, Phys. Rev. D 58 (1998) 084004 [INSPIRE].

[68] D. Ayzenberg, K. Yagi and N. Yunes, Linear Stability Analysis of Dynamical Quadratic Gravity, Phys. Rev. D 89 (2014) 044023 [arXiv:1310.6392] [InSPIRE].

[69] J.L. Blázquez-Salcedo et al., Quasinormal modes of compact objects in alternative theories of gravity, Eur. Phys. J. Plus 134 (2019) 46 [arXiv:1810.09432] [inSPIRE]. 
[70] M. Kimura, A simple test for stability of black hole by S-deformation, Class. Quant. Grav. 34 (2017) 235007 [arXiv: 1706.01447] [INSPIRE].

[71] M. Kimura and T. Tanaka, Robustness of the S-deformation method for black hole stability analysis, Class. Quant. Grav. 35 (2018) 195008 [arXiv:1805.08625] [INSPIRE].

[72] M. Kimura and T. Tanaka, Stability analysis of black holes by the S-deformation method for coupled systems, Class. Quant. Grav. 36 (2019) 055005 [arXiv: 1809.00795] [InSPIRE].

[73] D. Astefanesei, R.B. Mann and R. Rojas, Hairy Black Hole Chemistry, JHEP 11 (2019) 043 [arXiv: 1907.08636] [INSPIRE].

[74] A. Chamblin, R. Emparan, C.V. Johnson and R.C. Myers, Holography, thermodynamics and fluctuations of charged AdS black holes, Phys. Rev. D 60 (1999) 104026 [hep-th/9904197] [INSPIRE].

[75] A. Chamblin, R. Emparan, C.V. Johnson and R.C. Myers, Charged AdS black holes and catastrophic holography, Phys. Rev. D 60 (1999) 064018 [hep-th/9902170] [InSPIRE].

[76] A. Anabalon, D. Astefanesei, A. Gallerati and M. Trigiante, New non-extremal and BPS hairy black holes in gauged $\mathcal{N}=2$ and $\mathcal{N}=8$ supergravity, arXiv:2012.09877 [INSPIRE]. 